\title{
Bounds for quantities of interest and adaptivity in the Element Free Galerkin method
}

\author{
Yolanda Vidal, Núria Parés, Pedro Díez*and Antonio Huerta, \\ Departament de Matemàtica Aplicada III, Laboratori de Càlcul Numèric (LaCàN), Universitat Politècnica \\ de Catalunya, Jordi Girona 1, E-08034 Barcelona, Spain.
}

\section{SUMMARY}

A novel approach for implicit residual-type error estimation in mesh-free methods and an adaptive refinement strategy are presented. This allows computing upper and lower bounds of the error in energy norm with the ultimate goal of obtaining bounds for outputs of interest. The proposed approach precludes the main drawbacks of standard residual type estimators circumventing the need of fluxequilibration and resulting in a simple implementation that avoids integrals on edges/sides of a domain decomposition (mesh). This is especially interesting for mesh-free methods. The adaptive strategy proposed leads to a fast convergence of the bounds to the desired precision.

KEY WORDS: Element Free Galerkin, Mesh-free, Error estimation, Engineering outputs, Functional outputs, Goal-oriented error estimation, Residual based estimators, Adaptivity

\section{Introduction}

Assessment of functional outputs of the solution (goal-oriented error estimation) in computational mechanics problems is a real need in standard engineering practice. End-users of finite elements, finite differences or mesh-free codes are interested in obtaining bounds for quantities of engineering interest. Using a proper error representation, the bounds in the quantities of interest are obtained from bounds of the energy norm. Actually, these bounds are recovered combining upper and lower bounds of the energy error for both the original problem (primal) and an adjoint problem (associated with the selected functional output) [1, 2, 3, 4].

It is also important to note that bounds for the energy and for quantities of interest are usually obtained with respect to a reference solution (associated with a much larger space of approximation and a much finer discretization). Bounds for the exact solution of the boundary value problem as presented in $[5,6,7,8,9]$ are not addressed here.

The need of obtaining reliable upper and lower bounds of the error for quantities of interest has motivated the use of implicit residual error estimators, which are currently the only type

\footnotetext{
*Correspondence to: Campus Nord UPC, Módulo C-2, Jordi Girona 1, E-08034 Barcelona, Spain
} 
of estimators ensuring bounds for the error. Classical residual type estimators, which provide upper bounds of the error, require flux-equilibration procedures (hybrid-flux techniques) to properly set boundary conditions for local problems [10,2]. Flux-equilibration requires a domain decomposition, which is natural in finite elements but not in mesh-free methods. Moreover, it is performed by a complex algorithm, strongly dependent on the finite element type and requiring a data structure that is not natural in a standard finite element code.

The idea of using flux-free estimators, based on the partition-of-the-unity concept and using local subdomains different than elements, has been already proposed in $[11,12,13,14]$ for finite elements. The main advantage of the flux-free approach is the simplicity in the implementation. Obviously, this is especially important in the 3D case.

From the mesh-free point of view, another advantage is the fact that the local subdomains where the error equation is solved are the support of the functions characterizing the partition of unity. This is a concept that also exists in mesh-free methods and thus the extension is possible. Moreover, boundary conditions of the local problems are natural and the usual data structure of a code is directly employed.

In the last few years, some research has been devoted to develop error estimation procedures for mesh-free methods. Duarte and Oden [15] derive an explicit residual error estimator for the $h-p$ cloud method. Liu et al. [16] use a wavelet solution as an error indicator in an algorithm where multi-scale adaptive refinement is introduced. Chung and Belytschko [17] adapt the FEM stress projection technique for error analysis in Element Free Galerkin (EFG). Gavete et al. [18] propose a recovery-based-like error estimate presenting the standard drawbacks of these

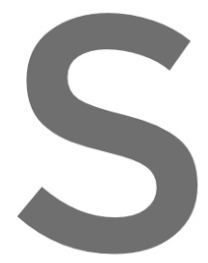

methods. None of $\mathrm{t}$ for functional outputs

To the authors mesh-free method Generalized Finite mesh-free methods ar
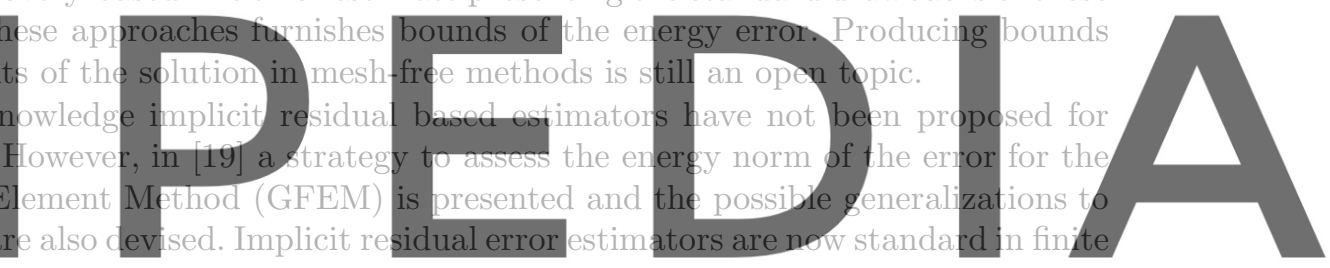

elements because they are mathematically sound, more accurate and allow computing upper

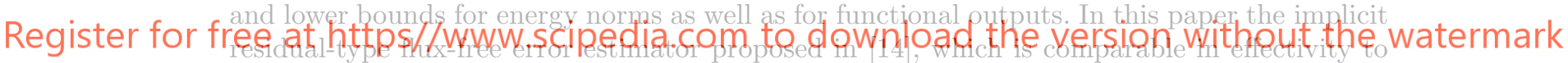

the standard hybrid-flux estimators, is extended to the Element Free Galerkin Method. In the present work, the difficulties found when extending the implicit residual methods to mesh-free are related with the fact that the refined discretization do not induce nested functional spaces. This is specially important for the error representation. Moreover, the bounds for the specific quantity of interest must be in this case carefully computed. In reference [19] these difficulties are not encountered because the error estimator is performed in terms of the energy norm and in the GFEM the refined subspaces are nested.

Mesh-free methods are especially well suited for adaptivity. Enriching the mesh-free discretization is straightforward because the new particles are added without any connectivity restriction. Here, a refinement scheme based on inserting particles following a quadtree structure in a background rectangular grid is proposed. The refinement criterion uses the information provided by the flux-free error estimator adapted for mesh-free methods.

The remainder of the paper is structured as follows. Section 2, following [14], recalls the basics on goal oriented error estimation in finite elements and recalls the basis of a fluxfree error estimator. Section 3 is devoted to extend step by step the previous concepts to the Element Free Galerkin method: definition of the reference error, estimation of outputs of 
interest, upper bound estimate of the energy norm of the reference error and, finally, bounds for the output. Section 4 deals with computational aspects related to numerical integration, Dirichlet boundary conditions and alternative bounds for the output. The adaptive strategy is introduced in Section 5. Finally, in Section 6, the performance of the proposed error estimator and adaptive strategy is tested in four numerical examples.

\section{Basics on goal oriented error estimation in FEM}

This section recalls basic results and strategies in the context of error estimation for linear outputs (quantities of interest) for finite elements. These strategies are extended to mesh-free methods in the following sections.

\subsection{Model Problem}

Let $\Omega \subset \mathbb{R}^{n_{\text {sd }}}$ be an open, bounded domain with piecewise linear boundary $\partial \Omega$ and $n_{\text {sd }}$ the number of spatial dimensions. The strong form of the problem reads: find $u$ such that

$$
\left\{\begin{aligned}
-\nabla^{2} u+\sigma u & =s & & \text { in } \Omega, \\
u & =u_{D} & & \text { on } \partial \Omega .
\end{aligned}\right.
$$

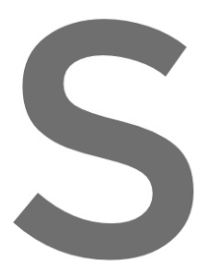

Only Dirichlet bo

to account also for conceptual difficul
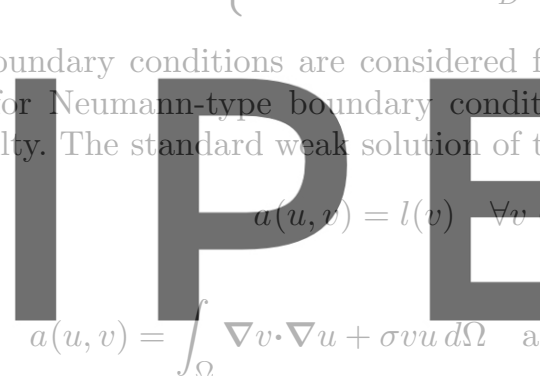

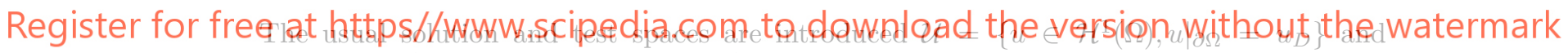
$\mathcal{W}_{0}=\left\{v \in \mathcal{H}^{1}(\Omega),\left.v\right|_{\partial \Omega}=0\right\}$, where $\mathcal{H}^{1}$ is the standard Sobolev space of square integrable functions and first derivatives. The bilinear form $a(\cdot, \cdot)$ induces the energy norm, which is denoted by $\|\cdot\|$, that is, $\|v\|^{2}=a(v, v)$.

The finite element interpolation spaces $\mathcal{U}^{H} \subset \mathcal{U}$ and $\mathcal{W}_{0}^{H} \subset \mathcal{W}_{0}$ are associated with a finite element mesh of characteristic size $H$ and degree $p$ for the complete interpolation polynomial base. The geometric support of the elements for a given mesh are open subdomains denoted by $\Omega_{k}, k=1 \ldots \mathrm{n}_{\mathrm{e} 1}$, where $\bar{\Omega}=\bigcup_{k} \bar{\Omega}_{k}$. It is also assumed that different elements do not overlap, that is, $\Omega_{k} \cap \Omega_{l}=\emptyset$ for $k \neq l$. Then, the finite element solution $u_{H}$ which is an approximation to $u$, lies in the finite dimensional space $\mathcal{U}^{H}$ and verifies

$$
a\left(u_{H}, v\right)=l(v) \quad \forall v \in \mathcal{W}_{0}^{H} .
$$

\subsection{Error equations and reference error}

The goal of a posteriori error estimation is to assess the accuracy of the finite element solution $u_{H}$, that is, to evaluate and measure the error, $e:=u-u_{H}$, which belongs to $\mathcal{W}_{0}$, either in the energy norm $\|e\|$ or in a quantity of interest as it is shown next. The equation for the error 
is recovered from (2) replacing the exact solution $u$ by $u_{H}+e$ and using the linearity of the first argument of $a(\cdot, \cdot)$

$$
a(e, v)=l(v)-a\left(u_{H}, v\right)=: R^{P}(v) \quad \forall v \in \mathcal{W}_{0},
$$

where $R^{P}(\cdot)$ stands for the weak residual associated to the finite element approximation $u_{H}$.

The exact error $e$ is replaced by a reference error, $e_{h}$, lying in a finite dimensional space $\mathcal{W}_{0}^{h}$ much richer than the original finite element space $\mathcal{W}_{0}^{H}$, i.e. $\mathcal{W}_{0}^{H} \subset \mathcal{W}_{0}^{h} \subset \mathcal{W}_{0}$. That is, the exact solution $u$ is replaced by the reference (or truth) solution $u_{h}$; consequently, $u \approx u_{h}=u_{H}+e_{h}$. The reference error $e_{h}$ can also be introduced as the projection of the exact error into the reference space. That is, $e_{h} \in \mathcal{W}_{0}^{h}$ is the solution of the problem

$$
a\left(e_{h}, v\right)=R^{P}(v) \quad \forall v \in \mathcal{W}_{0}^{h} .
$$

Direct evaluation of $e_{h}$ is computationally unaffordable because the size of the system of equations is the dimension of $\mathcal{W}_{0}^{h}$. The idea behind any implicit residual error estimator is to solve local problems instead of the global problem (4). Each of these local problems require proper boundary conditions in order to obtain a good approximation of the error and to ensure solvability.

\subsection{Estimation of outputs of interest}

The actual interest is to bound the output quantity $l^{\mathcal{O}}(u)$, where $l^{\mathcal{O}}(\cdot)$ is a linear functional
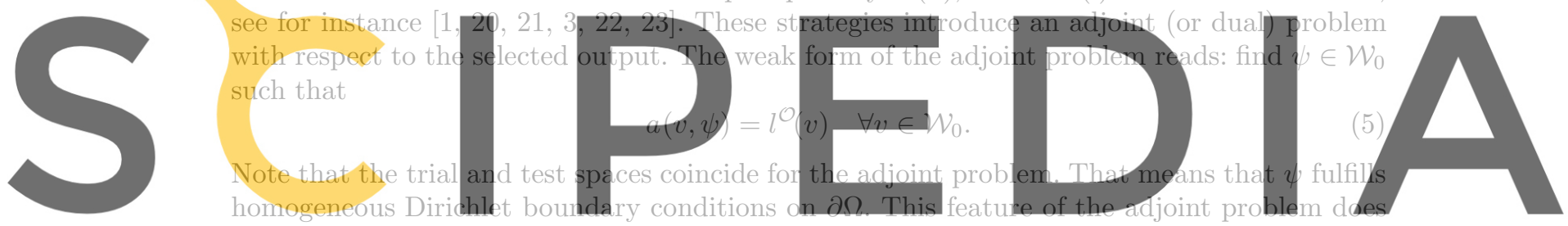

homogeneous Diridhlet boundary conditions on $2 \Omega$. This feature of the adjoint problem ddes

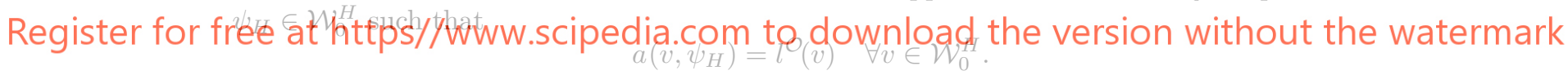

Finally, the adjoint reference error is $\epsilon_{h}:=\psi_{h}-\psi_{H} \in \mathcal{W}_{0}^{h}$, such that

$$
a\left(v, \epsilon_{h}\right)=l^{\mathcal{O}}(v)-a\left(v, \psi_{H}\right)=: R^{D}(v) \quad \forall v \in \mathcal{W}_{0}^{h},
$$

where $R^{D}$ is the weak residual associated with $\psi_{H}$.

If $v$ is replaced by $e_{h}$ in (6), then using Galerkin orthogonality $-a\left(e_{h}, v\right)=R^{P}(v)=0 \forall v \in$ $\mathcal{W}_{0}^{H}$ - and the parallelogram identity, the following representation of $l^{\mathcal{O}}\left(e_{h}\right)$ can be obtained

$$
l^{\mathcal{O}}\left(e_{h}\right)=a\left(e_{h}, \epsilon_{h}\right)=\frac{1}{4}\left\|\kappa e_{h}+\frac{1}{\kappa} \epsilon_{h}\right\|^{2}-\frac{1}{4}\left\|\kappa e_{h}-\frac{1}{\kappa} \epsilon_{h}\right\|^{2}
$$

for any non-zero arbitrary scalar parameter $\kappa$. To simplify the notation the arguments in the squared norms of the r.h.s. in (7) are denoted by $z_{h}^{ \pm}=\kappa e_{h} \pm \frac{1}{\kappa} \epsilon_{h}$.

In fact, in order to bound the output of the error, $l^{\mathcal{O}}\left(e_{h}\right)$, the r.h.s of $(7)$ indicates that it is sufficient to bound the energy norm of $z_{h}^{+}$and $z_{h}^{-}$, (i.e. the energy norm of linear combinations of $e_{h}$ and $\epsilon_{h}$ ). The notation $\left\|z_{h}^{ \pm}\right\|_{\mathrm{LB}}^{2}$ and $\left\|z_{h}^{ \pm}\right\|_{\mathrm{UB}}^{2}$ is used for estimates of $\left\|z_{h}^{ \pm}\right\|^{2}$ providing lower and upper bounds of the actual value of $\left\|z_{h}^{ \pm}\right\|^{2}$. This notation is used also in the following 
remainder with the same rationale. Note that the LB and UB subscripts in the norm are not intending to refer to different norms than $\|\cdot\|$. In fact, in practice $\left\|z_{h}^{ \pm}\right\|_{\text {UB }}^{2}$ is computed as the norm $\|\cdot\|$ applied to an estimate of the error function.

Once the bounds for $\left\|z_{h}^{ \pm}\right\|^{2}$ are computed, namely $\left\|z_{h}^{ \pm}\right\|_{\mathrm{LB}}^{2} \leq\left\|z_{h}^{ \pm}\right\|^{2} \leq\left\|z_{h}^{ \pm}\right\|_{\mathrm{UB}}^{2}$, the output of the error is readily bounded as

$$
\frac{1}{4}\left\|z_{h}^{+}\right\|_{\mathrm{LB}}^{2}-\frac{1}{4}\left\|z_{h}^{-}\right\|_{\mathrm{UB}}^{2} \leq l^{\mathcal{O}}\left(e_{h}\right) \leq \frac{1}{4}\left\|z_{h}^{+}\right\|_{\mathrm{UB}}^{2}-\frac{1}{4}\left\|z_{h}^{-}\right\|_{\mathrm{LB}}^{2},
$$

and, obviously, the bounds for the output of interest of the reference approximation, $l^{\mathcal{O}}\left(u_{h}\right)$, are

$$
l^{\mathcal{O}}\left(u_{H}\right)+\frac{1}{4}\left\|z_{h}^{+}\right\|_{\mathrm{LB}}^{2}-\frac{1}{4}\left\|z_{h}^{-}\right\|_{\mathrm{UB}}^{2} \leq l^{\mathcal{O}}\left(u_{h}\right) \leq l^{\mathcal{O}}\left(u_{H}\right)+\frac{1}{4}\left\|z_{h}^{+}\right\|_{\mathrm{UB}}^{2}-\frac{1}{4}\left\|z_{h}^{-}\right\|_{\mathrm{LB}}^{2} .
$$

Note that, in fact, since $\|v\|^{2} \geq 0,\left\|z_{h}^{ \pm}\right\|_{\mathrm{LB}}^{2}$ may be set to zero, yielding the well-known bounds for the output

$$
l^{\mathcal{O}}\left(u_{H}\right)-\frac{1}{4}\left\|z_{h}^{-}\right\|_{\mathrm{UB}}^{2} \leq l^{\mathcal{O}}\left(u_{h}\right) \leq l^{\mathcal{O}}\left(u_{H}\right)+\frac{1}{4}\left\|z_{h}^{+}\right\|_{\mathrm{UB}}^{2} .
$$

Therefore, it suffices to compute upper bounds of the energy norm of $z_{h}^{ \pm}$to recover bounds for $l^{\mathcal{O}}\left(u_{h}\right)$.

Next section introduces a methodology to obtain upper bound error estimates in energy norm. This approach is then used to compute $\left\|z_{h}^{+}\right\|_{\mathrm{UB}}^{2},\left\|z_{h}^{-}\right\|_{\mathrm{UB}}^{2}$.
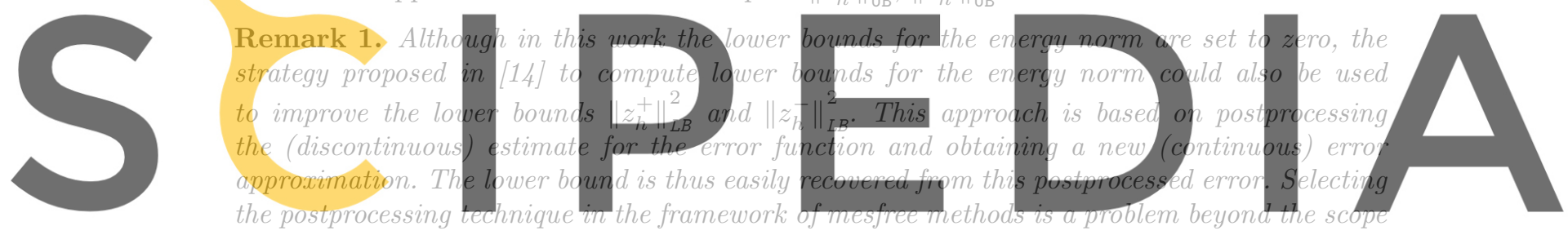

of this paper and requires further numerical testing. Consequently, in the following the lower

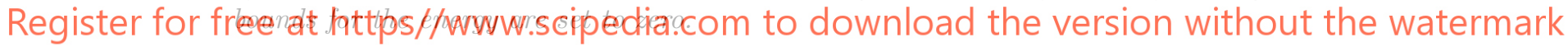

\subsection{A flux-free estimator yielding upper bounds of the error energy norm}

Let $\boldsymbol{x}^{i}, i=1, \ldots, \mathrm{n}_{\mathrm{np}}$ denote the nodes of the elements in the computational mesh (thus linked to $\mathcal{U}^{H}$ ) and $\phi^{i}$ the corresponding linear (or bilinear or trilinear) shape functions, which are such that $\phi^{i}\left(\boldsymbol{x}^{j}\right)=\delta_{i j}$. The support of $\phi^{i}$ is denoted by $\omega^{i}$ and it is called the star centered in, or associated with, node $\boldsymbol{x}^{i}$.

It is important to recall that the linear shape functions based on the nodes are a partition of unity. Using this essential property and the linearity of the weak residual $R^{P}(\cdot)$, defined in (3), the residual is decomposed into local contributions over each star

$$
R^{P}(v)=R^{P}\left(\sum_{i=1}^{\mathrm{n}_{\mathrm{np}}} \phi^{i} v\right)=\sum_{i=1}^{\mathrm{n}_{\mathrm{np}}} R^{P}\left(\phi^{i} v\right) \quad \forall v \in \mathcal{H}^{1}(\Omega)
$$

Note that $R^{P}\left(\phi^{i} v\right)$ vanishes if $\operatorname{supp} v \cap \omega^{i}=\emptyset$, since $\omega^{i}$ is the support of $\phi^{i}$.

The strategy to compute upper bound estimates of the reference error, $\left\|e_{h}\right\|_{\mathrm{UB}}^{2}$, consist in, first, evaluate the finite element solution $u_{H}$, which is necessary to compute the residual $R^{P}$. 
In a second phase the local estimates $e_{\omega^{i}}^{e s t} \in \mathcal{W}_{\omega^{i}}^{h}$, where $\mathcal{W}_{\omega^{i}}^{h}:=\mathcal{W}_{0}^{h} \cap \mathcal{H}^{1}\left(\omega^{i}\right)$, are defined as the solution of the following problems in each star $\omega^{i}$

$$
a_{\omega^{i}}\left(e_{\omega^{i}}^{e s t}, v\right)=R^{P}\left(\phi^{i} v\right) \quad \forall v \in \mathcal{W}_{\omega^{i}}^{h},
$$

where $a_{\omega^{i}}(\cdot, \cdot)$ is the restriction of the bilinear form $a(\cdot, \cdot)$ to the star $\omega^{i}$.

Remark 2. Formally any function $v \in \mathcal{W}_{\omega^{i}}^{h}$ is not defined in the whole domain $\Omega$ but only in the star $\omega^{i}$. However, here every $v \in \mathcal{W}_{\omega^{i}}^{h}$ is naturally extended to $\Omega$ by setting its value outside $\omega^{i}$ equal to zero. Thus, functions in $\mathcal{W}_{\omega^{i}}^{h}$ are continuous in $\omega^{i}$ but generally discontinuous across the boundary of the star.

Remark 3. The local restriction $\mathcal{W}_{0}^{h}$ to the element $\Omega_{k}, \mathcal{W}_{\Omega_{k}}^{h}:=\mathcal{W}_{0}^{h} \cap \mathcal{H}^{1}\left(\Omega_{k}\right)$, is also extended to $\Omega$ in the same way. This induces the broken space, namely

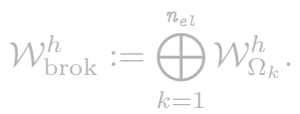

The bilinear form $a(\cdot, \cdot)$ and the energy norm are generalized to accept broken functions in its arguments; that is, for $v$ and $w \in \mathcal{W}_{\mathrm{brok}}^{h}$,

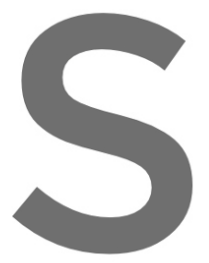

$$
a(v, w):=\sum^{n_{e l}} a_{\Omega_{k}}(v, w) \text { and }\|v\|^{2}:=\sum^{n_{e l}}\|v\|_{k}^{2}
$$
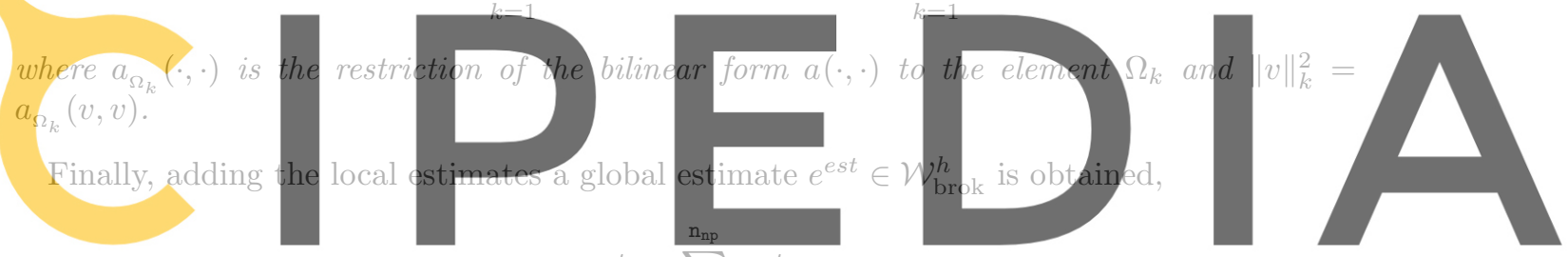

$$
e^{e s t}:=\sum e_{\omega^{i}}^{e s t}
$$

Register for free at https//www.scipedia.com to đ̛̣̊nload the version without the watermark and the upper bound of the energy norm of the reference error is recovered computing the norm of the estimate $e^{\text {est }}$. See [14] for a detailed description, development and formal analysis (viz. the proof of the next theorem) of this estimator.

Theorem 1. The estimate $e^{e s t}=\sum_{i=1}^{n_{n p}} e_{\omega^{i}}^{e s t} \in \mathcal{W}_{\mathrm{brok}}^{h}$ is such that

$$
a\left(e^{e s t}, v\right)=R^{P}(v) \quad \forall v \in \mathcal{W}_{0}^{h},
$$

and therefore

$$
\left\|e^{e s t}\right\|^{2} \geq\left\|e_{h}\right\|^{2}
$$

\section{Extension to Element Free Galerkin}

\subsection{Error equations and reference error}

Similarly to finite elements, in mesh-free methods a finite dimensional space $\mathcal{V}^{H} \subset \mathcal{H}^{1}(\Omega)$ is associated with a particle distribution of characteristic size $H$ and degree $p$ for the 
reproducibility imposed. Note however, that the functions in $\mathcal{V}^{H}$ do not generally vanish on $\partial \Omega$, since the boundary conditions are weakly imposed. In this case the solution and test spaces coincide and the Dirichlet boundary conditions are enforced using Nitche's method as it is discussed in the following.

Thus, the mesh-free solution $u_{H}$, which is an approximation to $u$, belongs to $\mathcal{V}^{H}$ and verifies

$$
a\left(u_{H}, v\right)=l(v) \quad \forall v \in \mathcal{V}^{H} .
$$

As in most error estimation strategies for finite elements, the exact solution $u$ is replaced by a reference (or truth) solution $u_{h}$, where $u_{h}$ is the Galerkin approximation of $u$ in a much finer space. Here, a reference space associated with a denser particle distribution $\mathcal{V}^{h}(h<<H)$ is considered, that is, $u_{h} \in \mathcal{V}^{h}$ is such that

$$
a\left(u_{h}, v\right)=l(v) \quad \forall v \in \mathcal{V}^{h} .
$$

However, contrary to in the FE context, in mesh-free methods the refined spaces are, in general. not nested, i.e. $\mathcal{V}^{H} \not \subset \mathcal{V}^{h}$. Therefore, in general, $u_{h}-u_{H}$ does not belong to $\mathcal{V}^{h}$.

The reference error in EFG is directly defined as the projection of the exact error into the reference space, i.e. $e_{h} \in \mathcal{V}^{h}$ is the solution of

$$
a\left(e_{h}, v\right)=R^{P}(v) \quad \forall v \in \mathcal{V}^{h},
$$

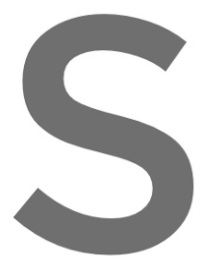

and, in general, $e_{h} \neq u_{h}-u_{H}$. This weak problem is very similar to (4) and only differs in
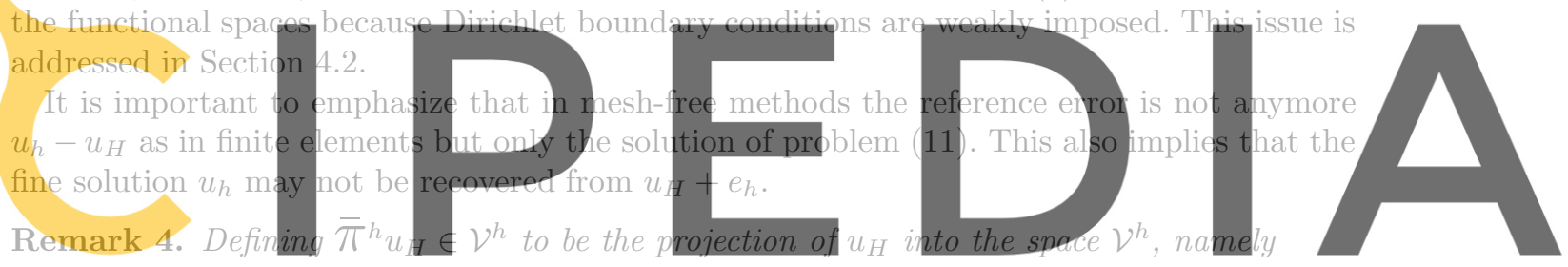

$$
a\left(\bar{\pi}^{h} u_{H}, v\right)=a\left(u_{H}, v\right) \quad \forall v \in v^{h}
$$

Register for free at https//www.scipedia.com to download the version without the watermark

$$
e_{h}=u_{h}-\bar{\pi}^{h} u_{H}
$$

or equivalently that $u_{h}=\bar{\pi}^{h} u_{H}+e_{h}$. Moreover, if the spaces $\mathcal{V}^{H}$ and $\mathcal{V}^{h}$ are nested, $\mathcal{V}^{H} \subset \mathcal{V}^{h}$ then $\pi^{h} u_{H}=u_{H}$.

\subsection{Estimation of outputs of interest}

Analogously to finite elements an adjoint problem is introduced to obtain a proper error representation, see (5). The adjoint problem consists in finding $\psi \in \mathcal{V}$ such that

$$
a(v, \psi)=l^{\mathcal{O}}(v) \quad \forall v \in \mathcal{V} .
$$

The corresponding EFG approximation and reference error are $\psi_{H} \in \mathcal{V}^{H}$ and $\epsilon_{h} \in \mathcal{V}^{h}$ such that

$$
a\left(v, \psi_{H}\right)=l^{\mathcal{O}}(v) \quad \forall v \in \mathcal{V}^{H}
$$

and

$$
a\left(v, \epsilon_{h}\right)=l^{\mathcal{O}}(v)-a\left(v, \psi_{H}\right)=: R^{D}(v) \quad \forall v \in \mathcal{V}^{h}
$$


Note again, that also in the adjoint problem the definition of the reference error is such that $\epsilon_{h} \neq \psi_{h}-\psi_{H}$, where the reference solution $\psi_{h}$ is defined analogously to $u_{h}$.

Replacing $v$ by $e_{h}$ in (15) yields

$$
l^{\mathcal{O}}\left(e_{h}\right)=a\left(e_{h}, \epsilon_{h}\right)+a\left(e_{h}, \psi_{H}\right) .
$$

Note that $e_{h} \in \mathcal{V}^{h}$ but not to $\mathcal{V}^{H}$ and therefore in general $a\left(e_{h}, \psi_{H}\right) \neq 0$.

An alternative error representation for the output $l^{\mathcal{O}}\left(e_{h}\right)$ is found using the parallelogram identity, see (7), in the first term of the r.h.s of (16)

$$
l^{\mathcal{O}}\left(e_{h}\right)=\frac{1}{4}\left\|\kappa e_{h}+\frac{1}{\kappa} \epsilon_{h}\right\|^{2}-\frac{1}{4}\left\|\kappa e_{h}-\frac{1}{\kappa} \epsilon_{h}\right\|^{2}+a\left(e_{h}, \psi_{H}\right) .
$$

As already mentioned and contrary to the finite element case, where the Galerkin orthogonality property and the fact that the spaces are nested guarantee $a\left(e_{h}, \psi_{H}\right)=0$, in the EFG context this is no longer valid. However, using the definition of the projection of $u_{H}$ into $\nu^{h}$, see remark 4 , and the Galerkin orthogonality property, $\nu^{H}$ is included in the kernel of $R^{P}$, this term is rewritten as,

$$
a\left(e_{h}, \psi_{H}\right)=a\left(e_{h}, \bar{\pi}^{h} \psi_{H}\right)=R^{P}\left(\bar{\pi}^{h} \psi_{H}\right)=R^{P}\left(\bar{\pi}^{h} \psi_{H}-\psi_{H}\right) .
$$

This expression clarifies the fact that the term $a\left(e_{h}, \psi_{H}\right)$ is asymptotically negligible in front of $a\left(e_{h}, \epsilon_{h}\right)$ as $h$ tends to 0 . Note that as $h \rightarrow 0, e_{h}$ and $\epsilon_{h}$ tend to the actual errors of $u_{H}$ and $\psi_{H}$, but they do not go to zero. On the contrary, $\bar{\pi}^{h} \psi_{H}-\psi_{H}$ goes to zero as $h$ decreases.

Thus, neglecting $a\left(e_{h}, \psi_{H}\right)$ and replacing the energy norms in equation (17) by the
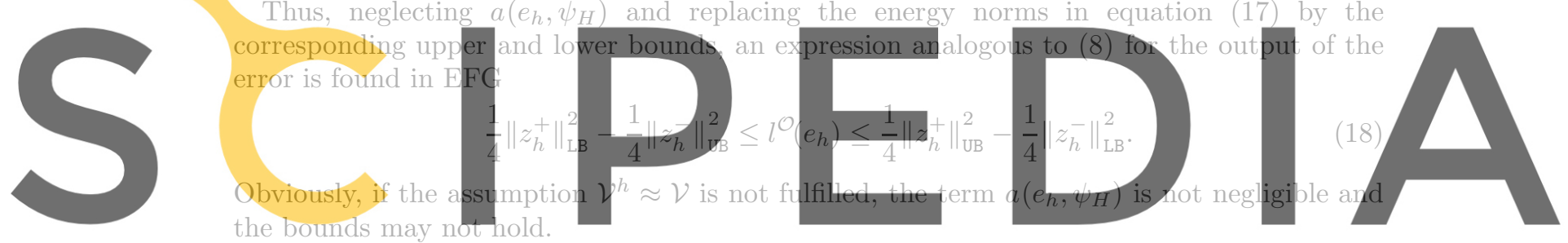

Recall that $e_{h} \neq u_{h}-u_{H}$. Consequently, it is not straightforward to obtain bounds of $l^{\mathcal{O}}\left(u_{h}\right)$

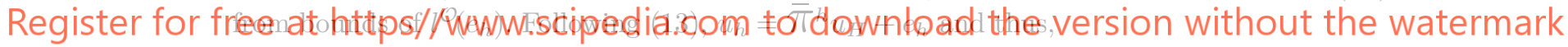

$$
l^{\mathcal{O}}\left(u_{h}\right)=l^{\mathcal{O}}\left(\bar{\pi}^{h} u_{H}\right)+l^{\mathcal{O}}\left(e_{h}\right)=l^{\mathcal{O}}\left(u_{H}\right)+l^{\mathcal{O}}\left(e_{h}\right)+l^{\mathcal{O}}\left(\bar{\pi}^{h} u_{H}-u_{H}\right)
$$

The third term in the r.h.s of (19) has the same behavior as the neglected term $a\left(e_{h}, \psi_{H}\right)$ in (18). The term $\bar{\pi}^{h} u_{H}-u_{H}$ goes to 0 as $h$ decreases and it is asymptotically negligible. That is, with the assumption used previously

$$
l^{\mathcal{O}}\left(u_{h}\right)=l^{\mathcal{O}}\left(u_{H}\right)+l^{\mathcal{O}}\left(e_{h}\right) .
$$

This allows obtaining bounds of $l^{\mathcal{O}}\left(u_{h}\right)$ from equation (18):

$$
l^{\mathcal{O}}\left(u_{H}\right)+\frac{1}{4}\left\|z_{h}^{+}\right\|_{\mathrm{LB}}^{2}-\frac{1}{4}\left\|z_{h}^{-}\right\|_{\mathrm{UB}}^{2} \leq l^{\mathcal{O}}\left(u_{h}\right) \leq l^{\mathcal{O}}\left(u_{H}\right)+\frac{1}{4}\left\|z_{h}^{+}\right\|_{\mathrm{UB}}^{2}-\frac{1}{4}\left\|z_{h}^{-}\right\|_{\mathrm{LB}}^{2} .
$$

In a nutshell, bounds for the output of the reference solution $u_{h}$ are derived in the EFG approach using the same expression than in the FE approach. (9) and (20) are identical. Thus, bounds for the output may be derived from upper bounds on the energy norm of $z_{h}^{ \pm}$, and may be enhanced adding lower bounds for the energy norm of $z_{h}^{ \pm}$. However, it is worth noting that in EFG these bounds are only valid under the assumption $\mathcal{V}^{h} \approx \mathcal{V}$ since the terms $a\left(e_{h}, \psi_{H}\right)$ and $l^{\mathcal{O}}\left(\bar{\pi}^{h} u_{H}-u_{H}\right)$ are neglected. 
Remark 5. An alternative approach precluding the drawback of assuming that $a\left(e_{h}, \psi_{H}\right)$ and $l^{\mathcal{O}}\left(\overline{\boldsymbol{\pi}}^{h} u_{H}-u_{H}\right)$ are negligible, but involving an extra computational cost is discussed in Section 4.3. Thus, it is possible to obtain bounds for the output of the reference approximation, $l^{\mathcal{O}}\left(u_{h}\right)$, which do not rely on the assumption $\mathcal{V}^{h} \approx \mathcal{V}$. Namely, they are valid regardless of the size of fine particle distribution. However, it is important to notice that in practice (for all studied examples) these extra terms are negligible.

\subsection{Extending flux-free error estimates to mesh-free methods}

In this section, error estimates yielding upper bounds of the energy norm are presented. For the sake of simplicity, the presentation concerns only the primal problem. The methodology is general and it is also applicable to the adjoint problem or to linear combinations of both. In particular, this methodology is applied to $z_{h}^{ \pm}$to obtain the required upper bounds for $\left\|z_{h}^{ \pm}\right\|^{2}$.

In EFG the upper estimate of the reference error can also be introduced with the same rationale as in finite elements. The partition of unity is naturally induced by the moving least squares interpolating functions, which are also denoted as $\phi^{i}$ ( $i$ now being the index of each particle)

In FE, the star associated with a node, $\omega_{i}$, is the support of the approximating function $\phi^{i}$. However, in the EFG context, in order to drastically reduce the computational effort, it is more convenient to take the star $\omega_{i}$ as the smallest integration sub-grid that includes the support of $\phi^{i}$. Figure 1 illustrates the definition of the star $\omega^{i}$. Let us denote now by $\Omega_{k}, k=1 \ldots \mathrm{n}_{\mathrm{cel}}$
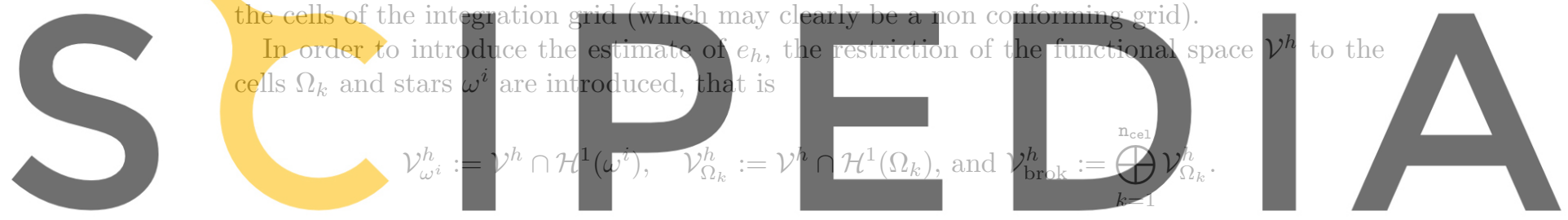

Note that the only difference with the local spaces defined in the FE context is that functions

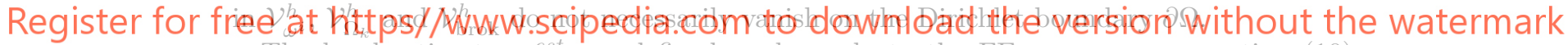

The local estimates $e_{s i_{i}}^{\text {est }}$ are defined analogously to the FE case - see equation (10)-.

Namely, $e_{\omega^{i}}^{e s t} \in \mathcal{V}_{\omega^{i}}^{h}$ are determined solving the local problems in each star $\omega^{i}$

$$
a_{\omega^{i}}\left(e_{\omega^{i}}^{e s t}, v\right)=R^{P}\left(\phi^{i} v\right) \quad \forall v \in \mathcal{V}_{\omega^{i}}^{h} .
$$

Recall that a local problem is solved in each star and that $e_{\omega^{i}}^{e s t}$ is extended (equal to zero) outside of $\omega^{i}$.

Defining the global estimate

$$
e^{e s t}:=\sum_{i=1}^{\mathrm{n}_{\mathrm{np}}} e_{\omega^{i}}^{e s t},
$$

it follows that

$$
a\left(e^{e s t}, v\right)=R^{P}(v) \quad \forall v \in \mathcal{V}^{h},
$$

an upper bound of the energy norm of the reference error is recovered computing the norm of the estimate $e^{\text {est }}$, namely

$$
\left\|e^{e s t}\right\|^{2} \geq\left\|e_{h}\right\|^{2} .
$$




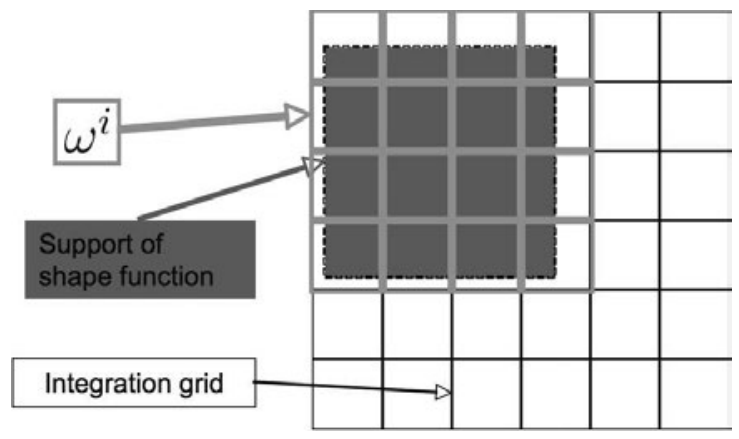

Figure 1. Definition of the star $\omega^{i}$.

This result follows from a trivial extension of theorem 1 where now the reference error, $e_{h}$, and the local estimates, $e_{\omega^{i}}^{\text {est }}$, do not necessarily vanish on the Dirichlet boundary $\partial \Omega$. Note that the bilinear form $a(\cdot, \cdot)$ and, hence, the energy norm $\|\cdot\|$ include in the EFG context terms associated with the boundary, see Section 4.2.

Remark 6. The solvability of the local problem, equation (21), is ensured because for an arbitrary function $w$ the kernel of $a_{\omega^{i}}(w, \cdot)$ includes only constant functions. Thus, for $v$ constant the r.h.s of (21) is zero due to the Galerkin orthogonality, i.e. $R^{P}\left(\phi^{i} v\right)=0$ for
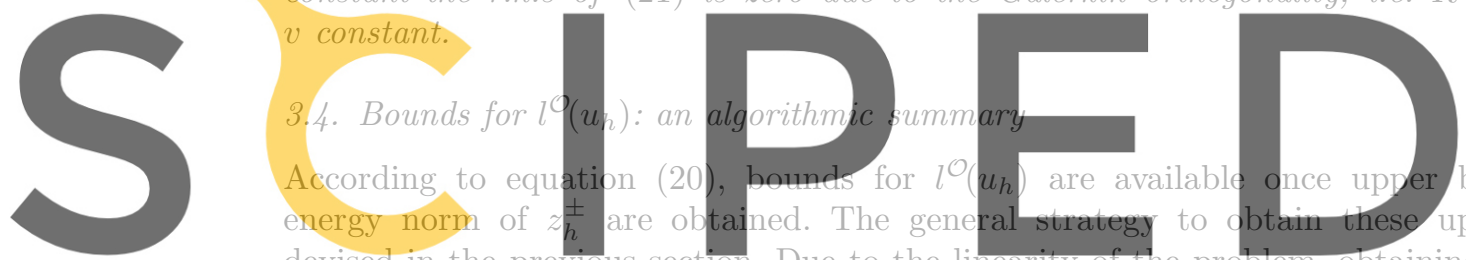

for $z_{h}^{ \pm}$is equivalent to obtain the estimates for the primal and adjoint problem, that is

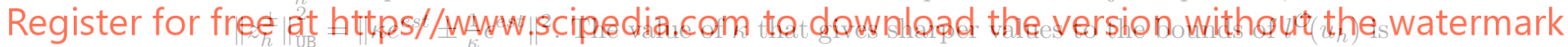

the one that minimizes $\left\|z_{h}^{ \pm}\right\|_{\text {UR }}^{2}$. This optimal value (see [21]) is $\kappa^{2}=\left\|\epsilon^{e s t}\right\| /\left\|e^{e s t}\right\|$.

The main steps of the procedure to compute bounds for $l^{O}\left(u_{h}\right)$ is summarized in Figure 2, for the optimal choice of the parameter $\kappa$.

\section{Computational aspects}

\subsection{Numerical integration}

The coarse (global) problem is solved with a given numerical quadrature. The numerical approximations of the linear and bilinear forms $l(\cdot)$ and $a(\cdot, \cdot)$ using this quadrature are denoted by $l_{Q}(\cdot)$ and $a_{Q}(\cdot, \cdot)$. In practice the approximate solution $u_{H}$ is such that,

$$
a_{Q}\left(u_{H}, v\right)=l_{Q}(v) \quad \forall v \in \mathcal{V}^{H} .
$$

Then, as expected, the associated residual is zero for functions in the test space:

$$
R_{Q}^{P}(v)=l_{Q}(v)-a_{Q}\left(u_{H}, v\right)=0 \quad \forall v \in \mathcal{V}^{H} .
$$


Step 1.- Compute $u_{H}$ and $\psi_{H} \in \mathcal{V}^{H}$.

Step 2.- For $i=1, \ldots, \mathrm{n}_{\mathrm{np}}$, compute the local estimates $e_{\omega^{i}}^{e s t}$ and $\epsilon_{\omega^{i}}^{e s t} \in \mathcal{V}_{\omega^{i}}^{h}$ such that

$$
a_{\omega^{i}}\left(e_{\omega^{i}}^{e s t}, v\right)=R^{P}\left(\phi^{i} v\right), \quad a_{\omega^{i}}\left(v, \epsilon_{\omega^{i}}^{e s t}\right)=R^{D}\left(\phi^{i} v\right) \quad \forall v \in \mathcal{V}_{\omega^{i}}^{h}
$$

Step 3.- Define the global estimates $e^{e s t}$ and $\epsilon^{e s t} \in \mathcal{V}_{\mathrm{brok}}^{h}$ as

$$
e^{e s t}=\sum_{i=1}^{\mathrm{n}_{\mathrm{np}}} e_{\omega^{i}}^{e s t}, \quad \epsilon^{e s t}=\sum_{i=1}^{\mathrm{n}_{\mathrm{np}}} \epsilon_{\omega^{i}}^{e s t} .
$$

Step 4.- Compute the upper bounds of the energy norm of $z_{h}^{ \pm}$as

$$
\left\|z_{h}^{ \pm}\right\|_{\mathrm{UB}}^{2}:=2\left\|e^{e s t}\right\|\left\|\epsilon^{e s t}\right\| \pm 2 a\left(e^{e s t}, \epsilon^{e s t}\right) \geq\left\|z_{h}^{ \pm}\right\|^{2}
$$

Step 5.- Recover the bounds for the output $l^{\mathcal{O}}\left(u_{h}\right)$ as

$$
l^{\mathcal{O}}\left(u_{H}\right)-\frac{1}{4}\left\|z_{h}^{-}\right\|_{\mathrm{UB}}^{2} \leq l^{\mathcal{O}}\left(u_{h}\right) \leq l^{\mathcal{O}}\left(u_{H}\right)+\frac{1}{4}\left\|z_{h}^{+}\right\|_{\mathrm{UB}}^{2}
$$

Figure 2. Bounds for the output $l^{\mathcal{O}}\left(u_{h}\right)$

However, for a different (finer) quadrature,

$$
a_{q}\left(u_{H}, v\right) \neq l_{q}(v) \quad \forall v \in \mathcal{V}^{H},
$$

and, thus, Galerkin orthogonality is no longer valid with the richer quadrature,

$$
R_{q}^{P}(v)=l_{q}(v)-a_{q}\left(u_{H}, v\right) \neq 0 \quad \forall v \in \mathcal{V}^{H}
$$

Recall that Galerkin orthogonality is needed both to proof the upper bound property and to ensure solvability of the local problems, see remark 6. Consequently, the same quadrature must be used to obtain the approximate solution $u_{H}\left(\right.$ in $\mathcal{V}^{H}$ ) and to solve the local problems $(21)$ (in $\left.\mathcal{V}^{h}\right)$. This obviously implies that integration in the "coarse" computation is performed with a quadrature accurate enough for the reference discretization. This is obviously an extra cost required to assess the error distribution.

\subsection{Dirichlet boundary conditions}

In the mesh-free context, shape functions do not fulfill the Kronecker delta property. Therefore, imposing Dirichlet boundary conditions is not as simple as for finite elements. In recent years, many specific techniques for the implementation of essential boundary conditions in meshfree methods have been developed. A general overview on existing techniques is presented in [24]. Amongst the different techniques discussed in [24] the continuous blending method $[25,26,27]$ (i.e. introduce standard finite elements along the boundary and adapt the meshfree interpolation functions to obtain completeness) and Nitsche's method [28, 29, 30] are the most suitable alternatives. Here, it is assumed in the formulation that Nitsche's method is used. This results in a modified version of the weak form and a test space without any boundary restriction. 
Using the Nitsche's method the weak form of problem (1) is find $u \in \mathcal{H}^{1}(\Omega)$ such that

$$
a(u, v)=l(v) \quad \forall v \in \mathcal{H}^{1}(\Omega),
$$

where

$$
\begin{gathered}
a(u, v)=\int_{\Omega} \boldsymbol{\nabla} v \cdot \nabla u+\sigma v u d \Omega \\
\quad-\int_{\partial \Omega} v \boldsymbol{n} \cdot \nabla u d \Gamma-\int_{\partial \Omega} u \boldsymbol{n} \cdot \boldsymbol{\nabla} v d \Gamma+\beta \int_{\partial \Omega} v u d \Gamma, \\
l(v)=\int_{\Omega} v s d \Omega-\int_{\partial \Omega} u_{D} \boldsymbol{n} \cdot \boldsymbol{\nabla} v d \Gamma+\beta \int_{\partial \Omega} v u_{D} d \Gamma .
\end{gathered}
$$

Note that equation (23) is identical to (2) but the linear and bilinear operators and the related functional spaces are different. Note also that this bilinear form $a(\cdot, \cdot)$ is also symmetric and positive definite and, hence, induces a norm. In fact, the last term in (24) is required to ensure coercivity of the bilinear form $a(\cdot, \cdot)$ provided that $\beta$ is large enough. The parameter $\beta$ must be larger than a minimum value that can be assessed as $\gamma / \ell$, where $\ell$ is a characteristic size of the discretization (say $H$ or $h$ for the discretizations mentioned here), and $\gamma$ is a constant that can be obtained as the maximum eigenvalue of some eigenvalue problem [31]. In practice that means that $\beta$ must increase when the discretization gets finer.

In order to keep the same bilinear form both in the computational and the reference discretization, the value of $\beta$ is selected with the criteria associated with the fine discretization and used for both cases, analogously as for the numerical quadrature.

\subsection{Alternative bounds for the output}

As mentioned in remark 5 it is possible to obtain bounds for the output of the reference approximation, $l^{\mathcal{O}}\left(u_{h}\right)$, which do not rely on the assumption $\mathcal{V}^{h} \approx \mathcal{V}$. That is, without neglecting the terms $l^{\mathcal{O}}\left(\overline{\boldsymbol{T}}^{h} u_{H}-u_{H}\right)$ and $a\left(e_{h}, \psi_{H}\right)$. Namely, they are valid regardless of the size of the coarse and fine discretizations.

The key idea is to replace $u_{H}$ by a weaker projection of the solution $u_{H}$ into the fine space $\mathcal{V}^{h}$. Let $\hat{u}_{H} \in \mathcal{V}^{h}$ be an approximation of $u_{H}$ such that,

$$
a\left(\hat{u}_{H}, v\right)=a\left(u_{H}, v\right) \quad \forall v \in \mathcal{V}^{H} .
$$

It is worth noting that the projection $\hat{u}_{H}$ is not uniquely determined by the previous equation. In practice (for all studied examples) $\hat{u}_{H}$ is selected among the solutions of (26) as the element in $\mathcal{V}^{h}$ such that the euclidean norm of the vector of its nodal values is minimum.

Taking $\hat{u}_{H} \in \mathcal{V}^{h}$ as an approximation of $u_{H}$, a modified reference error can be defined,

$$
\hat{e}_{h}:=u_{h}-\hat{u}_{H} \in \mathcal{V}^{h},
$$

where $\hat{e}_{h} \in \mathcal{V}^{h}$ verifies

$$
a\left(\hat{e}_{h}, v\right)=l(v)-a\left(\hat{u}_{H}, v\right):=\hat{R}^{P}(v) \quad \forall v \in \mathcal{V}^{h} .
$$

Equation (27) gives a linear expression similar to FE, and it is straightforward to provide bounds for $l^{\mathcal{O}}\left(u_{h}\right)$ from bounds of $l^{\mathcal{O}}\left(\hat{e}_{h}\right)$. The remainder of this section is devoted to describe how to find these bounds and to analyze their behavior. 
It is noteworthy that orthogonality of the modified residual with respect to the space $\mathcal{V}^{H}$ is verified

$$
\hat{R}^{P}(v)=l(v)-a\left(\hat{u}_{H}, v\right)=l(v)-a\left(u_{H}, v\right)=0 \quad \forall v \in \mathcal{V}^{H} .
$$

Therefore, the error estimation procedure described in Section 3.3 can be applied to find $\hat{e}^{e s t} \in \mathcal{V}_{\text {brok }}^{h}$ such that

$$
a\left(\hat{e}^{e s t}, v\right)=\hat{R}^{P}(v) \quad \forall v \in \mathcal{V}^{h},
$$

yielding to $\left\|\hat{e}^{e s t}\right\|^{2} \geq\left\|\hat{e}_{h}\right\|^{2}$, that is, the norm of $\hat{e}^{e s t}$ is an upper bound of the energy norm of the reference error $\hat{e}_{h}$.

Proceeding similarly for the adjoint problem, we introduce $\hat{\psi}_{H} \in \mathcal{V}^{h}$ such that,

$$
a\left(v, \hat{\psi}_{H}\right)=a\left(v, \psi_{H}\right) \quad \forall v \in \mathcal{V}^{H},
$$

and the modified associated adjoint reference error $\hat{\epsilon}_{h}:=\psi_{h}-\hat{\psi}_{H}$, where $\hat{\epsilon}_{h} \in \mathcal{V}^{h}$ verifies

$$
a\left(v, \hat{\epsilon}_{h}\right)=l^{\mathcal{O}}(v)-a\left(v, \hat{\psi}_{H}\right):=\hat{R}^{D}(v) \quad \forall v \in \mathcal{V}^{h} .
$$

As for the primal problem, the adjoint residual is orthogonal to the space $\mathcal{V}^{H}$

$$
\hat{R}^{D}\left(v_{H}\right)=l^{\mathcal{O}}\left(v_{H}\right)-a\left(v_{H}, \hat{\psi}_{H}\right)=l^{\mathcal{O}}\left(v_{H}\right)-a\left(v_{H}, \psi_{H}\right)=0 .
$$

Therefore, the error estimation procedure described in Section 3.3 can be also applied to find $\hat{\epsilon}^{e s t} \in \mathcal{V}_{\text {brok }}^{h}$ such that

$$
a\left(v, \hat{\epsilon}^{e s t}\right)=\hat{R}^{D}(v) \quad \forall v \in \mathcal{V}^{h},
$$

yielding to $\left\|\hat{\epsilon}^{e s t}\right\|^{2} \geq\left\|\hat{\epsilon}_{h}\right\|^{2}$, that is, the norm of $\hat{\epsilon}^{e s t}$ is an upper bound of the energy norm of the dual reference error $\hat{\epsilon}_{h}$.

The estimates $\hat{e}^{e s t}$ and $\hat{\epsilon}^{\text {est }}$ allow to obtain strict bounds of $l^{\mathcal{O}}\left(u_{h}\right)$ as follows. First, from $(27)$

$$
u_{h}=\hat{u}_{H}+\hat{e}_{h}
$$

and the output can be written as,

$$
l^{\mathcal{O}}\left(u_{h}\right)=l^{\mathcal{O}}\left(\hat{u}_{H}\right)+l^{\mathcal{O}}\left(\hat{e}_{h}\right) .
$$

Second, using equation (30) with $v=\hat{e}_{h}$ and equation (28) with $v=\hat{u}_{H}$ and substituting in the previous equation $l^{\mathcal{O}}\left(u_{h}\right)$ can be expressed as,

$$
l^{\mathcal{O}}\left(u_{h}\right)=l^{\mathcal{O}}\left(\hat{u}_{H}\right)+a\left(\hat{e}_{h}, \hat{\epsilon}_{h}\right)+\hat{R}^{P}\left(\hat{\psi}_{H}\right) .
$$

Where now $\hat{R}^{P}\left(\hat{\psi}_{H}\right)$ is computable.

Applying the parallelogram identity to the term $a\left(\hat{e}_{h}, \hat{\epsilon_{h}}\right)$ and using the same rationale than in Section 2.3 the bounds for the output are obtained:

$$
l^{\mathcal{O}}\left(u_{h}\right) \geq l^{\mathcal{O}}\left(\hat{u}_{H}\right)+\hat{R}^{P}\left(\hat{\psi}_{H}\right)-\frac{1}{4}\left\|\kappa \hat{e}^{e s t}-\frac{1}{\kappa} \hat{\epsilon}^{e s t}\right\|^{2}
$$

and

$$
l^{\mathcal{O}}\left(u_{h}\right) \leq l^{\mathcal{O}}\left(\hat{u}_{H}\right)+\hat{R}^{P}\left(\hat{\psi}_{H}\right)+\frac{1}{4}\left\|\kappa \hat{e}^{e s t}+\frac{1}{\kappa} \hat{\epsilon}^{e s t}\right\|^{2} .
$$

This alternative strategy allows to bound the output of the reference approximation without neglecting any terms but with the extra computational cost of finding the projections $\hat{u}_{H}$ and $\hat{\psi}_{H}$. 


\section{Adaptive strategy}

In a finite element context, adaptive strategies consist on building a new mesh adapting the element size to the requirements of the solution. That is, reducing the element size where the interpolation must be enriched (i.e. where more accuracy is needed) and enlarging the elements where it is already accurate enough. The remeshing criterion is the expression that translates the output of the error estimator into a spatial distribution of the optimal element size for the new mesh, which is the input of the mesh generator.

The remeshing criterion takes as input the elementary error distribution and produces the information required to build up a new mesh. If the remeshing criterion is properly derived, the new mesh should provide an approximate solution satisfying the accuracy requirements at a minimum computational cost. From a practical viewpoint, the remeshing criterion is produced by a function that for each element of the mesh provides the size of the elements that will be located in the position of the former element.

In the EFG context, the particle distribution does not define a natural decomposition of the domain into non-overlapping subdomains where to compute the error contribution and where to predict the new size. However, the entire domain can be partitioned into nonoverlapping cells using the integration grid. Recall that the integration cells are denoted by $\Omega_{k}, k=1, \ldots, \mathrm{n}_{\mathrm{cel}}$ where $\mathrm{n}_{\mathrm{cel}}$ denotes the number of integration cells covering the entire domain. The size of each cell, $H_{k}:=\sqrt{\left|\Omega_{k}\right|}$, is assumed to be proportional to the interparticle distance in that cell, $H_{k}^{\text {ip }}$. That is, it exists a constant $\alpha$ (in the examples $\alpha=1 / 4$ ) such that

$$
H_{k}=\alpha H_{k}^{\text {ip }} .
$$

The adaptive strategy consists in first, the contribution of the local error for each integration cell, $\|e\|_{k}$ and $\|\epsilon\|_{k}$ must be determined. Then, use this local information to predict the new size of the integration cells that should be located in the position of the former cell. The new discretization is described by $\tilde{H}_{k}$ which is the desired cell size for all the new cells lying in cell $\Omega_{k}$ of the former discretization. Finally, the new interparticle distance for the particles of the new discretization is $\tilde{H}_{k}^{i p}:=\tilde{H}_{k} / \alpha$. The goal of this section is to find an expression giving $\tilde{H}_{k}$ as a function of the assessed error in $\Omega_{k}$, and such that the error in the new grid

$$
\tilde{e}=u-u_{\tilde{H}}
$$

fulfills an accuracy restriction of the type $\left|l^{\mathcal{O}}(\tilde{e})\right| \leq \Delta_{\text {tol }}$.

A key point of any adaptive strategy is to properly define the quantity that needs to be controlled. In standard adaptivity, the aim is to control the (squared) energy norm of the error, whereas in goal-oriented adaptivity the goal is to control $\left|l^{\mathcal{O}}(\tilde{e})\right|$. Obviously the resulting expressions for $\tilde{H}_{k}$ are different. Here, the adopted strategy is to control the output of the error $\left|l^{\mathcal{O}}(\tilde{e})\right|$ by controlling a conservative bound for $\left|l^{\mathcal{O}}(\tilde{e})\right|$. However, other options could be considered as in [32].

Indeed, using the definition of the adjoint problem, equation (14), the Galerkin orthogonality property of the exact error $\tilde{e}$ with respect to the test space $\mathcal{V}^{\tilde{H}}$ and the Cauchy-Schwartz inequality, the output $l^{\mathcal{O}}(\tilde{e})$ may be bounded by

$$
\left|l^{\mathcal{O}}(\tilde{e})\right|=|a(\tilde{e}, \psi)|=|a(\tilde{e}, \tilde{\epsilon})| \leq\|\tilde{e}\|\|\epsilon\|,
$$

where $\tilde{\epsilon}=\psi-\psi_{\tilde{H}}$ analogously to (32). This bound is less sharp than the parallelogram identity (7) but simpler. Moreover, this bound can be decomposed into local contributions on 
the integration cells as

$$
\|\tilde{e}\|\|\tilde{\epsilon}\|=\frac{1}{2}\left(\kappa^{2}\|\tilde{e}\|^{2}+\frac{1}{\kappa^{2}}\|\tilde{\epsilon}\|^{2}\right)=\sum_{\tilde{k}=1}^{\tilde{n}_{\text {ce1 }}} \frac{1}{2}\left(\kappa^{2}\|\tilde{e}\|_{\tilde{k}}^{2}+\frac{1}{\kappa^{2}}\|\tilde{\epsilon}\|_{\tilde{k}}^{2}\right),
$$

where analogously to remark 3 the local norm in the new cell $\tilde{\Omega}_{\tilde{k}}$ is $\|\cdot\|_{\tilde{k}}$ and $\kappa^{2}:=\|\tilde{\epsilon}\| /\|\tilde{e}\|$. In practice $\kappa$ is assumed to be independent of $H$, that is $\kappa^{2}=\|\epsilon\| /\|e\|$, see remark 7 .

Thus

$$
\left|l^{\mathcal{O}}(\tilde{e})\right| \leq\|\tilde{e}\|\|\epsilon\|=\sum_{\tilde{k}=1}^{\tilde{n}_{\text {cel }}} \frac{1}{2}\left(\kappa^{2}\|\tilde{e}\|_{\tilde{k}}^{2}+\frac{1}{\kappa^{2}}\|\tilde{\epsilon}\|_{\tilde{k}}^{2}\right)
$$

and, consequently, in order to ensure $\left|l^{\mathcal{O}}(\tilde{e})\right| \leq \Delta_{\text {tol }}$ it suffices that

$$
\frac{1}{2}\left(\kappa^{2}\|\tilde{e}\|_{\tilde{k}}^{2}+\frac{1}{\kappa^{2}}\|\tilde{\epsilon}\|_{\tilde{k}}^{2}\right) \leq \Delta_{\tilde{k}}
$$

with

$$
\sum_{\tilde{k}=1}^{\tilde{\mathrm{n}}_{\mathrm{cel}}} \Delta_{\tilde{k}}=\Delta_{t o l}
$$

Deriving the adaptive criterion requires some assumptions in the local convergence of the solutions:

- An a priori estimate for the error of the mesh-free approximation $u_{H}$ stands, see [33],

$$
\|e\| \leq C_{1} \rho^{p}\|u\|_{\mathcal{H}^{p}(\Omega)},
$$

where the constant $C_{1}$ does not depend on the dilation parameter $\rho$ and $\rho$ may be substituted by the interparticle distance $H^{\text {ip }}$ since $\rho / H^{\text {ip }}$ is kept constant.

- A local a priori error estimate (a particularization of the previous formula over one integration cell) is available if local optimality of the EFG approximation holds, that is,

$$
\|e\|_{k} \leq C_{2}\left\|u-v_{H}\right\|_{k} \quad \forall v_{H} \in \mathcal{V}^{H}\left(\Omega_{k}\right) .
$$

This assumption stands if pollution errors are negligible, which is a rather weak hypothesis in adaptive computation since the influence of pollution becomes negligible as the adaptive process progresses. Under the previous hypothesis a local a priori error estimate describing the asymptotic behavior of the local error norm in each integration cell can be written as

$$
\|e\|_{k} \leq C_{3}\left(H^{\text {ip }}\right)^{p}\|u\|_{\mathcal{H}^{p}\left(\Omega_{k}\right)} .
$$

- Assuming that the norm $\|u\|_{\mathcal{H}^{p}\left(\Omega_{k}\right)}$ is bounded by the global one

$$
\|u\|_{\mathcal{H}^{p}\left(\Omega_{k}\right)} \leq C_{5} \frac{\left|\Omega_{k}\right|^{1 / 2}}{|\Omega|^{1 / 2}}\|u\|_{\mathcal{H}^{p}(\Omega)},
$$

where the areas $\left|\Omega_{k}\right| \approx H_{k}^{2}$ and $|\Omega|$ are used to normalize the expressions, an a priori estimate is obtained from equation (35),

$$
\|e\|_{k} \leq C_{4}\left(H^{\mathrm{ip}}\right)^{p} H_{k} .
$$


Under the previous assumptions, the local a priori estimates for the primal and adjoint problems

$$
\|e\|_{k} \leq C_{u} H_{k}^{p+1} \quad \text { and } \quad\|\epsilon\|_{k} \leq C_{\psi} H_{k}^{p+1}
$$

are derived taking into account equation (31).

The same behavior is assumed to hold in the new discretization

$$
\|\tilde{e}\|_{\tilde{k}} \leq C_{u} \tilde{H}_{k}^{p+1} \quad \text { and } \quad\|\tilde{\epsilon}\|_{\tilde{k}} \leq C_{\psi} \tilde{H}_{k}^{p+1} .
$$

where the local measures $\|\cdot\|_{\tilde{k}}$ refer to cells of the new integration grid lying in $\Omega_{k}$.

The standard Richardson extrapolation is applied:

$$
\|\tilde{e}\|_{\tilde{k}} \approx \frac{\tilde{H}_{k}^{p+1}}{H_{k}^{p+1}}\|e\|_{k} \quad \text { and } \quad\|\tilde{\epsilon}\|_{\tilde{k}} \approx \frac{\tilde{H}_{k}^{p+1}}{H_{k}^{p+1}}\|\epsilon\|_{k} .
$$

Introducing this expressions into (33) yields the desired relation between the current cell size $H_{k}$ and the new ones $\tilde{H}_{k}$, namely

$$
\frac{1}{2}\left(\kappa^{2} \frac{\tilde{H}_{k}^{2(p+1)}}{H_{k}^{2(p+1)}}\|e\|_{k}^{2}+\frac{1}{\kappa^{2}} \frac{\tilde{H}_{k}^{2(p+1)}}{H_{k}^{2(p+1)}}\|\epsilon\|_{k}^{2}\right)=\Delta_{\tilde{k}},
$$

so that the new element size will be

$$
\tilde{H}_{k}^{2(p+1)}=\frac{\Delta_{\tilde{k}}}{\kappa^{2}\|e\|_{k}^{2}+\frac{1}{\kappa^{2}}\|\epsilon\|_{k}^{2}} 2 H_{k}^{2(p+1)} .
$$

To obtain a computable expression, the local energy norms $\|e\|_{k}$ and $\|\epsilon\|_{k}$ are replaced by its upper bound estimates $\left\|e^{e s t}\right\|_{k}$ and $\left\|\epsilon^{e s t}\right\|_{k}$ respectively. Also, the parameter $\kappa^{2}=\|\tilde{\epsilon}\| /\|\tilde{e}\|$ is approximated by $\left\|\epsilon^{e s t}\right\| /\left\|e^{e s t}\right\|$, namely

$$
\tilde{H}_{k}^{2(p+1)}=\frac{\Delta_{\tilde{k}}}{(\kappa)^{2}\left\|e^{e s t}\right\|_{k}^{2}+\frac{1}{(\kappa)^{2}}\left\|\epsilon^{e s t}\right\|_{k}^{2}} 2 H_{k}^{2(p+1)} .
$$

Finally, in order to set $\Delta_{k}$, an assumption must be done on the error distribution in the optimal grid. Here it is assumed that in an optimal grid, a uniform error density is attained, [34]. That is, the local contributions to the error must be proportional to the size of the cell

$$
\Delta_{\tilde{k}}=\Delta_{t o l} \frac{\tilde{H}_{k}^{2}}{|\Omega|} \text { for } \tilde{k} \text { such that } \tilde{\Omega}_{\tilde{k}} \subset \Omega_{k}
$$

The final expression to compute $\tilde{H}_{k}$ is

$$
\tilde{H}_{k}^{2 p}=\frac{\Delta_{t o l}}{\kappa^{2}\left\|e^{e s t}\right\|_{k}^{2}+\frac{1}{\kappa^{2}}\left\|\epsilon^{e s t}\right\|_{k}^{2}} \frac{2 H_{k}^{2(p+1)}}{|\Omega|} .
$$

Using the proposed adaptive strategy, the generation of the new particle distribution proceeds as follows:

1. For each integration cell, $\Omega_{k}$, the new integration cell size $\tilde{H}_{k}$ is computed.

2. Using relation (31) a new interparticle distance, $\tilde{H}_{k}^{\text {ip }}$, is predicted for the particles lying inside $\Omega_{k}$. 
3. The particle distribution is generated as the nodes of a non-conforming cartesian grid. This grid is recursively generated starting from a uniform grid of the whole domain $\Omega$ of grid size equal to the maximum interparticle distances $\tilde{H}_{k}^{\text {ip }}$. Then the cells of the uniform grid are refined using a quadtree strategy until the cell size is equal or smaller to the minimum particle distance that has to be imposed in the nodes of each cell.

Remark 7. Using the a priori estimates for the errors of the mesh-free primal and adjoint approximations $u_{H}$ and $\psi_{H}$ and taking in account that the constants of this estimates do not depend on the interparticle distance, one has

$$
\begin{aligned}
& \|e\| \leq C_{1} \rho^{p}\|u\|_{\mathcal{H}^{p}(\Omega)} \quad \text { and } \quad\|\tilde{e}\| \leq C_{1} \tilde{\rho}^{p}\|u\|_{\mathcal{H}^{p}(\Omega)}, \\
& \|\epsilon\| \leq C_{6} \rho^{p}\|\psi\|_{\mathcal{H}^{p}(\Omega)} \quad \text { and } \quad\|\tilde{\epsilon}\| \leq C_{6} \tilde{\rho}^{p}\|\psi\|_{\mathcal{H}^{p}(\Omega)} .
\end{aligned}
$$

Thus, it is straightforward that $\|\epsilon\| /\|e\|=\|\tilde{\epsilon}\| /\|\tilde{e}\|$. Once in the asymptotic range, $\kappa$ does not depend on the discretization.

Remark 8. Recall that in order to obtain a computable expression, the local energy norms $\|e\|_{k}$ and $\|\epsilon\|_{k}$ are replaced by its upper bound estimates $\left\|e^{\text {est }}\right\|_{k}$ and $\left\|\epsilon^{\text {est }}\right\|_{k}$ respectively. Note that replacing the energy norms of the exact errors by its estimates leads to,

$$
\sum_{\tilde{k}=1}^{\tilde{n}_{\text {cel }}} \frac{1}{2}\left(\kappa^{2}\left\|e^{e s t}\right\|_{k}^{2}+\frac{1}{\kappa^{2}}\left\|\epsilon^{e s t}\right\|_{k}^{2}\right) \leq \Delta_{t o l} .
$$

It is noteworthy that the l.h.s of previous expression is the half bound gap. This means that the proposed strategy (replacing the errors by their estimates) in fact predicts the new distribution in order to obtain a half bound gap less than the prescribed tolerance, $\Delta_{t o l}$. In a nutshell, the half bound gap represents where the proposed adaptive process actually refines the distribution.

\section{Numerical results}

This section includes four numerical examples illustrating the performance of the proposed error estimation procedure and the behavior of the adaptive strategy presented in Section 5 .

In the numerical tests presented in this section, the upper and lower bounds for the output $l^{\mathcal{O}}\left(u_{h}\right)$ are denoted by $\eta_{\mathrm{LB}}$ and $\eta_{\mathrm{UB}}$

$$
\eta_{\mathrm{LB}}:=l^{\mathcal{O}}\left(u_{H}\right)-\frac{1}{4}\left\|z_{h}^{-}\right\|_{\mathrm{UB}}^{2} \leq l^{\mathcal{O}}\left(u_{h}\right) \leq l^{\mathcal{O}}\left(u_{H}\right)+\frac{1}{4}\left\|z_{h}^{+}\right\|_{\mathrm{UB}}^{2}=: \eta_{\mathrm{UB}} .
$$

The bound average, $\eta_{\mathrm{AVE}}:=\left(\eta_{\mathrm{UB}}+\eta_{\mathrm{LB}}\right) / 2$, is taken as a new approximation of the quantity of interest, and the half bound gap, $\Delta=\left(\eta_{\mathrm{UB}}-\eta_{\mathrm{LB}}\right) / 2$, is seen as an error indicator (see remark 8).

The convergence of the bounds for the output is analyzed studying the bounds for $l^{\mathcal{O}}\left(e_{h}\right)$

$$
-\frac{1}{4}\left\|z_{h}^{-}\right\|_{\mathrm{UB}}^{2} \leq l^{\mathcal{O}}\left(e_{h}\right) \leq \frac{1}{4}\left\|z_{h}^{+}\right\|_{\mathrm{UB}}^{2} .
$$

The convergence of the error of the EFG approximations measured in the energy norm in the case $p=1$ is linear, and therefore, the expected convergence for $l^{\mathcal{O}}\left(e_{h}\right)$ is quadratic (since it involves squared norms of the primal and adjoint errors). 


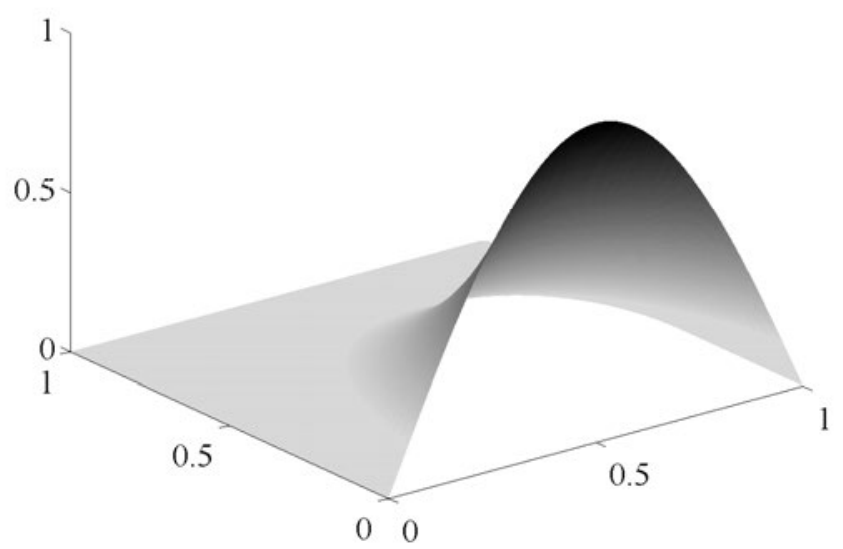

Figure 3. Example 1: analytical solution.

\subsection{Example 1: 2D Laplace equation}

The 2D Laplace problem $(\sigma=0$ and $s=0$ in $(1))$

$$
\left\{\begin{array}{rlrl}
\nabla^{2} u & =0 & & \text { in }(x, y) \in] 0,1[\times] 0,1[ \\
u(x, 0) & =\sin (\pi x) & & \\
u(x, 1) & =u(0, y)=u(1, y)=0 &
\end{array}\right.
$$

analyzed in [24] is considered. The analytical solution of the problem is (see Figure 3)

$$
u(x, y)=(\cosh (\pi y)-\operatorname{coth}(\pi) \sinh (\pi y)) \sin (\pi x) .
$$

The selected quantity of interest is the average of the solution over the domain,

$$
l^{\mathcal{O}}(u)=\int_{[0,1]^{2}} u d \Omega .
$$

The test is used to show different features of the proposed error estimator and adaptive strategy. First the example demonstrates that the terms that are not taken into account in the expression of the bounds for the output are indeed negligible. Second, the convergence of the bounds is shown using a uniform refinement of the particle distribution. Third, the behavior of the bounds with respect to an adaptive refinement is analyzed. Finally, the adaptive strategy is tested allowing also for unrefinement.

The influence of the different terms appearing in the representation for the output, $l^{\mathcal{O}}\left(u_{h}\right)$, is analyzed for a fixed coarse particle distribution of $11 \times 11$ particles $(H=0.1)$ and for a varying size of the reference particle distribution. Recall, that from equations (19) and (16)

$$
l^{\mathcal{O}}\left(u_{h}\right)=l^{\mathcal{O}}\left(u_{H}\right)+a\left(e_{h}, \epsilon_{h}\right)+a\left(e_{h}, \psi_{H}\right)+l^{\mathcal{O}}\left(\bar{\pi}^{h} u_{H}-u_{H}\right) .
$$

In order to derive the bounds for $l^{\mathcal{O}}\left(u_{h}\right)$, the last two terms of the r.h.s. are neglected assuming that $\mathcal{V}^{h} \approx \mathcal{V}$, and upper and lower bounds for $a\left(e_{h}, \epsilon_{h}\right)$ are computed. Table 6.1 shows the 


\begin{tabular}{|c|c|c|c|c|}
\hline$h$ & $l^{\mathcal{O}}\left(u_{H}\right)$ & $l^{\mathcal{O}}\left(u_{h}\right)$ & $a\left(e_{h}, \epsilon_{h}\right)$ & neglected terms \\
\hline$H / 4$ & \multirow{3}{*}{0.187345} & 0.186142 & $-1.120 \mathrm{E}-3$ & $-8.3 \mathrm{E}-5$ \\
\hline$H / 8$ & & 0.186034 & $-1.300 \mathrm{E}-3$ & $-1.0 \mathrm{E}-5$ \\
\hline$H / 16$ & & 0.185921 & $-1.426 \mathrm{E}-3$ & $-1.0 \mathrm{E}-6$ \\
\hline
\end{tabular}

Table I. Example 1: comparison of the terms appearing in the representation of $l^{\mathcal{O}}\left(u_{h}\right)$ given in equation (37) for a fixed coarse particle distribution of $11 \times 11$ particles $(H=0.1)$ and for a varying reference particle distribution.

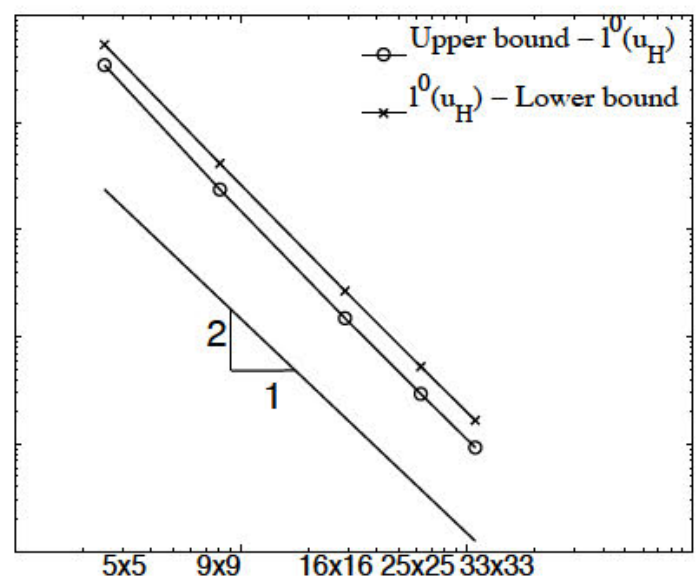

Figure 4. Example 1: convergence of the bounds for the error in the output $l^{\mathcal{O}}\left(e_{h}\right)$ for a uniform $H$-refinement of the particle distribution.

different terms appearing in (37), where the neglected terms refers to $a\left(e_{h}, \psi_{H}\right)+l^{\mathcal{O}}\left(\bar{\pi}^{h} u_{H}-\right.$ $\left.u_{H}\right)$, and where the reference particle distributions are obtained from the coarse distribution setting $h=H / 4, H / 8$ and $H / 16$. It can be seen that even in the case $h=H / 4$, the neglected terms are approximately $10 \%$ of $a\left(e_{h}, \epsilon_{h}\right)$. Moreover, it is worth noting that as the size of $h$ decreases, the neglected terms converge to zero whereas the terms $a\left(e_{h}, \epsilon_{h}\right)$ and $l^{\mathcal{O}}\left(u_{h}\right)$ stabilize to the value of $a(e, \epsilon)$ and $l^{\mathcal{O}}(u)$ respectively (the neglected terms are $\simeq 1 \%$ for $h=H / 8$ and $\simeq 0.1 \%$ for $h=H / 16$ ). Therefore, even for the case $h=H / 4$, the results are in good agreement with the assumption made in Section 3.2 that $\mathcal{V}^{h} \approx \mathcal{V}$. Although the alternative approach proposed in Section 4.3 could be employed, it will yield to nearly equal results and require an additional computational effort.

The convergence of the output bounds is analyzed for a uniform refinement of the particle distribution in a series of structured distributions of the particles. The initial particle distribution is composed of $5 \times 5$ particles $(H=1 / 4)$ and in each refinement step, the new particle distribution is obtained dividing by 2 the interparticle distance in each direction $\left(H_{n e w}=H / 2\right)$. The convergence of the upper and lower bounds for $l^{\mathcal{O}}\left(e_{h}\right)$ is shown in Figure 
\#par=25
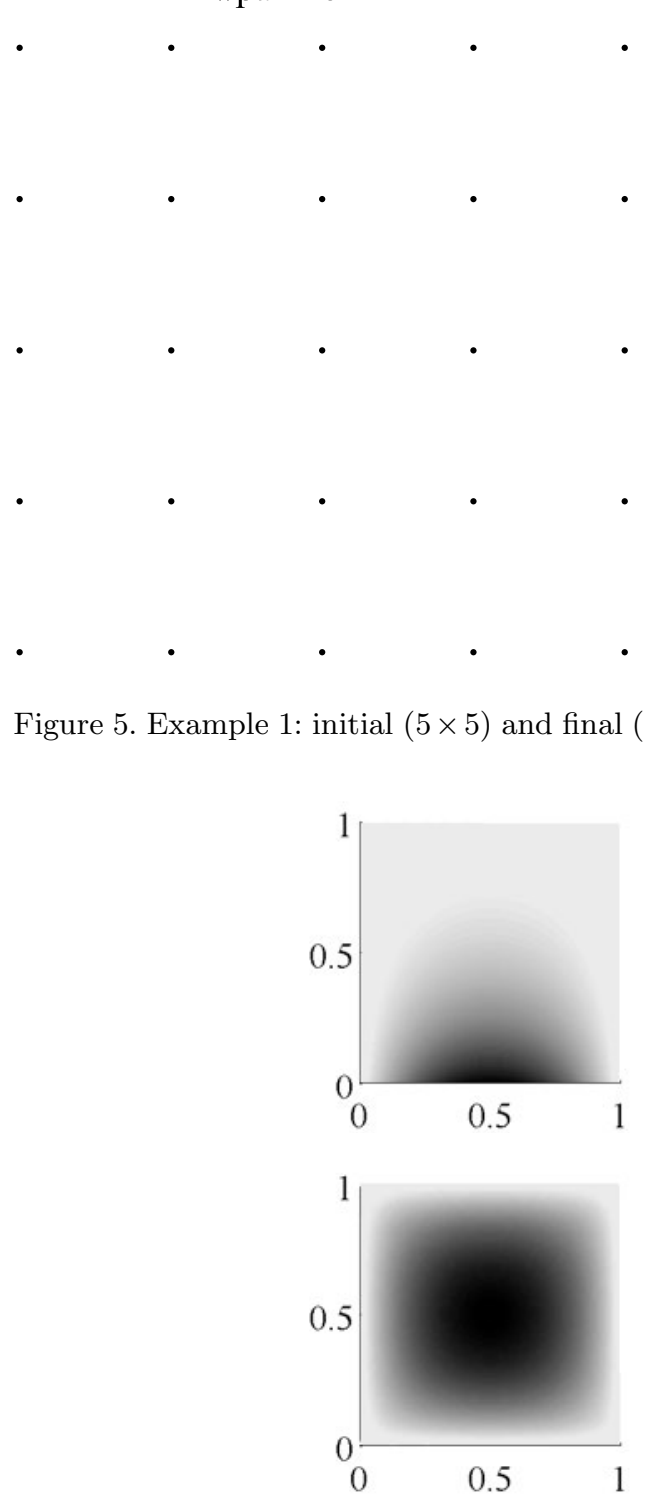

\#par=171

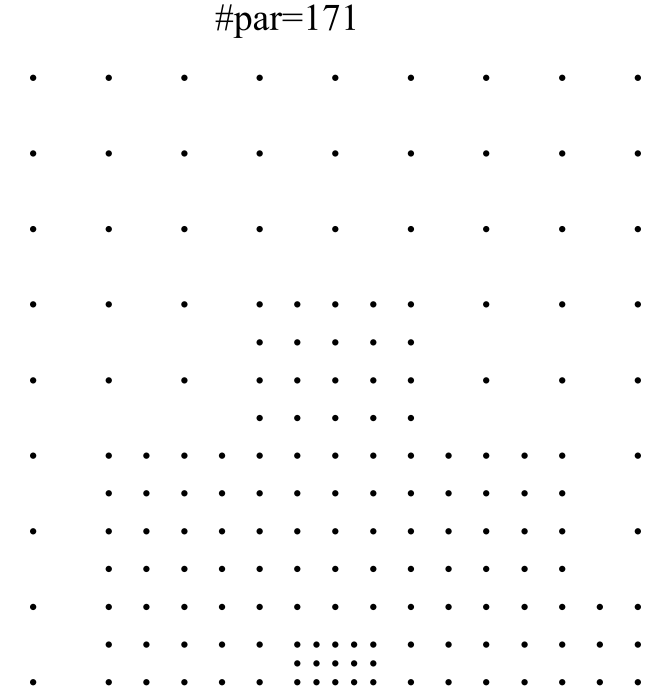




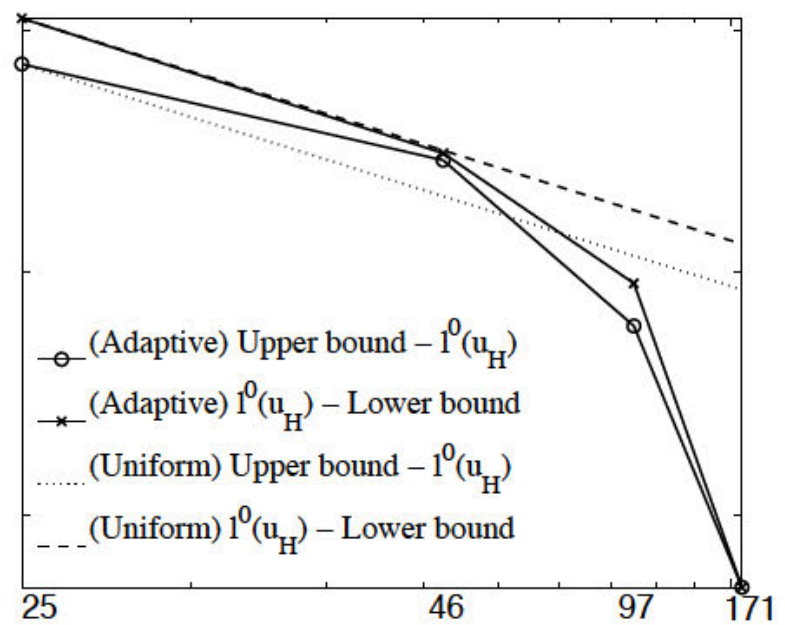

Figure 7. Example 1: convergence of the error estimates following both the uniform refinement strategy and the adaptive strategy.

particles (for $p=1$ and $h=H / 4$ ). The initial and final particle distributions are shown in Figure 5 and the primal and adjoint EFG approximations of $u$ and $\psi$ for the final particle distribution are represented in Figure 6. In Figure 7 the convergence behavior of a uniform refinement strategy is compared with the adaptive strategy. It is worth noting that both the upper bound estimates and the lower bound estimates show the standard behavior predicted by the a priori estimates in the uniform refinement and a much better convergence rate for the adapted refinement.

The results along the adaptive process are shown in Figure 8. The figure shows the evolution of both the upper and lower bounds for the output, the EFG approximation of the output and the exact value of the output. The bound average is also shown as a new approximation of the quantity of interest. For the initial coarse distribution of particles the output is bounded by, $l^{\mathcal{O}}\left(u_{h}\right) \in[0.1801,0.1932]$, that is

$$
l^{\mathcal{O}}\left(u_{h}\right)=0.1866 \pm 0.0066=0.1866 \pm 3.54 \%,
$$

while in the final particle distribution, the estimation procedure provides a much narrower interval, $l^{\mathcal{O}}\left(u_{h}\right) \in[0.1858,0.1859]$

$$
l^{\mathcal{O}}\left(u_{h}\right)=0.1859 \pm 6.5 \mathrm{E}-5=0.1859 \pm 0.035 \%,
$$

with a particle distribution of only 171 particles. The half bound gap is reduced from $3.54 \%$ to $0.035 \%$ with only three steps of the adaptive procedure. The local contributions of each integration cell $\Delta_{k}$ to the half bound gap are plotted in Figure 9 for the initial particle distribution. Since the local contributions to the half bound gap are the indicators used in the adaptive process, the algorithm refines the zones with larger values of the local contributions. Figure 9 also shows the spatial distribution of $\left|l^{\mathcal{O}}\left(e_{h}\right)\right|$ - in each integration cell, the value of the restriction of the integral defining $l^{\mathcal{O}}$ to cell $\Omega_{k},\left|l_{k}^{\mathcal{O}}\left(e_{h}\right)\right|$, is represented. This is in good agreement with the local indicators provided by the half bound gap. Although the optimal 


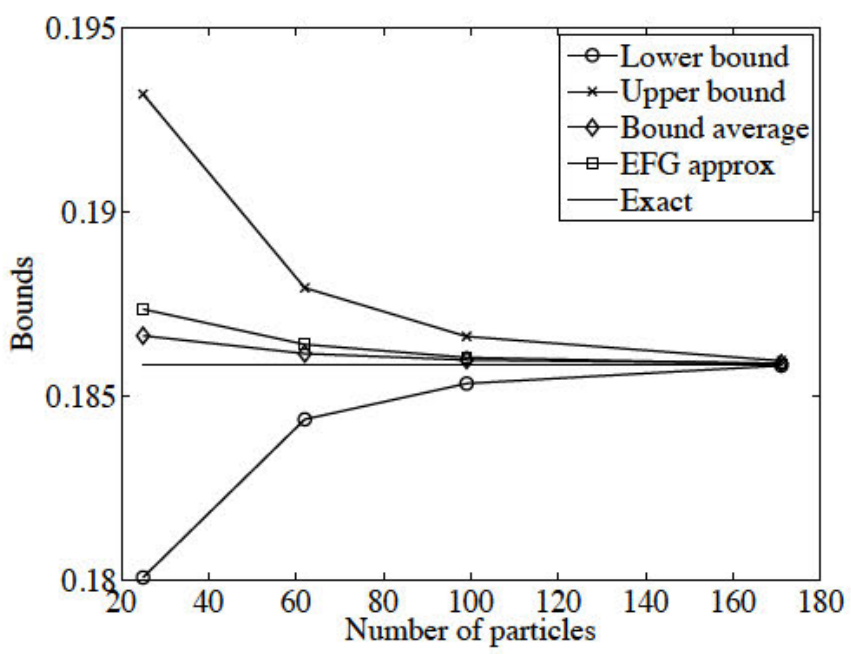

Figure 8. Example 1: estimates and bounds in the adaptive procedure.

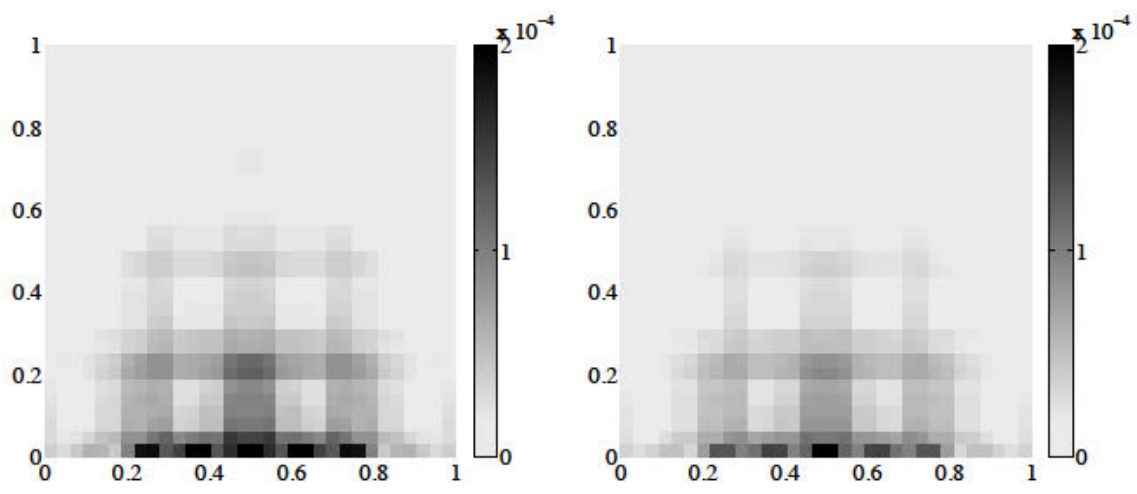

Figure 9. Example 1: spatial distribution of the half bound gap (left) and of $\left|l^{\mathcal{O}}\left(e_{h}\right)\right|$ (right).

refinement criterion should take into account the cancelations of the contributions $l_{k}^{\mathcal{O}}\left(e_{h}\right)$ to the total error $\left|l^{\mathcal{O}}\left(e_{h}\right)\right|$, a safer approach is considered here. The adaptive strategy aims at minimizing the local contributions $\left|l_{k}^{\mathcal{O}}\left(e_{h}\right)\right|$ and the half bound gap is taken as an indicator for this local contributions.

The behavior of the error estimation procedure when assessing the energy norm is also analyzed both for the primal error $e_{h}$ and for the linear combinations of the primal and adjoint errors, $z_{h}^{ \pm}$. The local contributions to the upper bound for $\left\|e_{h}\right\|,\left\|e^{e s t}\right\|_{k}$, are shown both for the initial and final particle distributions in Figure 10. Although the adaptive procedure is oriented to reduce the error in the output, it also yields a good reduction to the error energy norm of the primal error. Figure 11 shows the spatial distribution of the effectivity indexes of $\left\|z_{h}^{+}\right\|_{\mathrm{UB}}^{2}$ and $\left\|z_{h}^{-}\right\|_{\mathrm{UB}}^{2}$. The local effectivity indexes are computed with respect to the exact value 
Local error

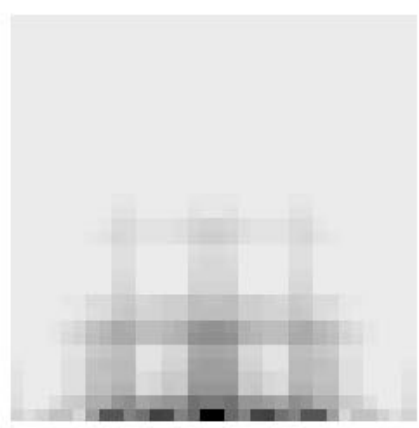

Local error

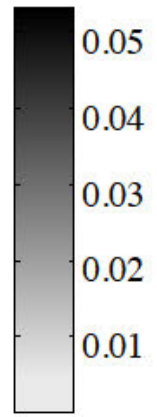

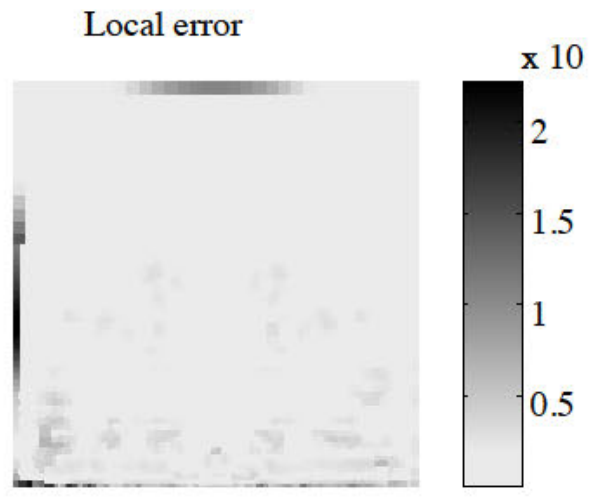

Figure 10. Example 1: local contributions to the upper bound of the energy norm of the primal error for the initial (left) and final (right) particle distribution of the adaptive $H$-refinement.
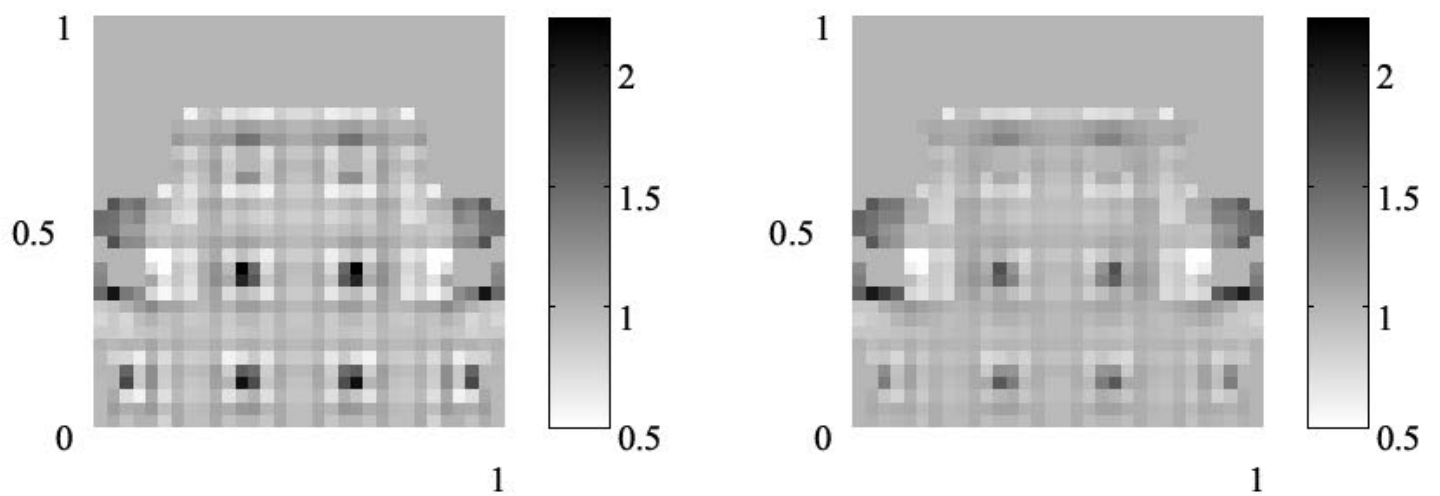

Figure 11. Example 1: effectivity indices of $\left\|z_{h}^{+}\right\|_{\mathrm{UB}}^{2}$ (left) and $\left\|z_{h}^{-}\right\|_{\mathrm{UB}}^{2}$ (right) respectively in the initial particle distribution of the adaptive $H$-refinement.

of the local norms, $\left\|z_{h}^{ \pm}\right\|_{k}^{2}$, that is

$$
\frac{\text { local cell contribution to }\left\|z_{h}^{ \pm}\right\|_{\mathrm{ub}}^{2}}{\left\|z_{h}^{ \pm}\right\|_{k}^{2}}
$$

Although the particle distribution is excessively coarse and the error is large, the effectivity indices shown for the upper bounds are always close to the optimum value of one. Since the local contributions to $\left\|z_{h}^{ \pm}\right\|_{\mathrm{UB}}^{2}$ are used to compute the indicators of the goal-oriented adaptive procedure (local half bound gap), is is important to obtain good effectivity indexes for $\left\|z_{h}^{ \pm}\right\|_{\mathrm{UB}}^{2}$.

Finally, the unrefinement capability of the adaptive strategy proposed in Section 5 is tested starting with an initial uniform distribution of 289 particles $(17 \times 17)$. Figure 12 shows that after one step of the adaptive strategy a new distribution with less particles than the initial one is obtained (241 particles). The distribution is coarsened in regions where the half bound 
\#par=289
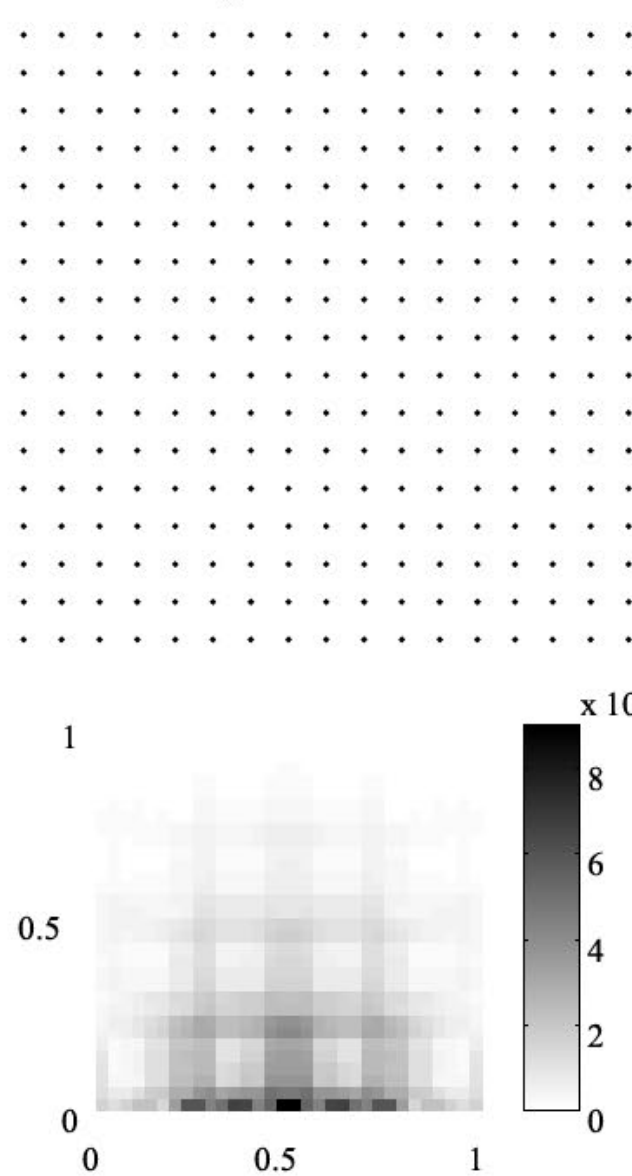

\#par=241

$-5$

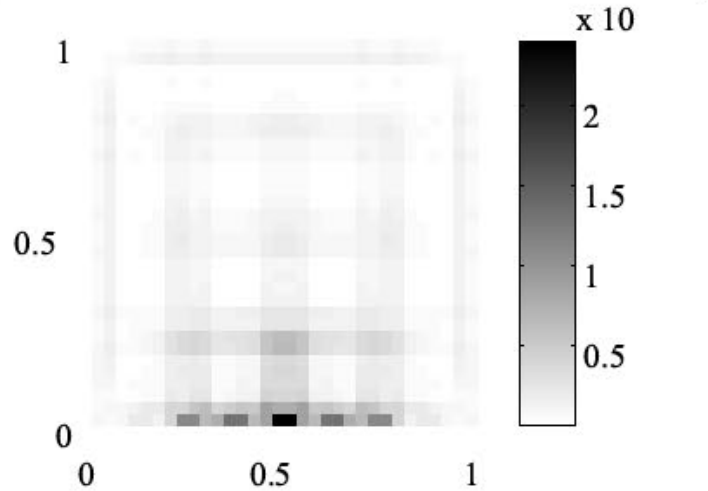

Figure 12. Example 1: refinement/unrefinement capability of the adaptive strategy.

gap is less than the desired tolerance and refined in regions where it is larger than the desired.

\subsection{Example 2: Poisson equation with exponential source term}

The second example is a well-known benchmark problem, see [2, 3, 35, 14]. Problem (1) is solved for $\sigma=0$ and $u_{D}=0$

$$
\left\{\begin{aligned}
-\nabla^{2} u=s & \text { in } \Omega \\
u=0 & \text { on } \partial \Omega
\end{aligned}\right.
$$

where the source term is chosen such that the exact solution has the following analytical expression (see Figure 13)

$$
u(x, y)=x^{2}(1-x)^{2}\left(e^{10 x^{2}}-1\right) y^{2}(1-y)^{2}\left(e^{10 y^{2}}-1\right) / 2000 .
$$




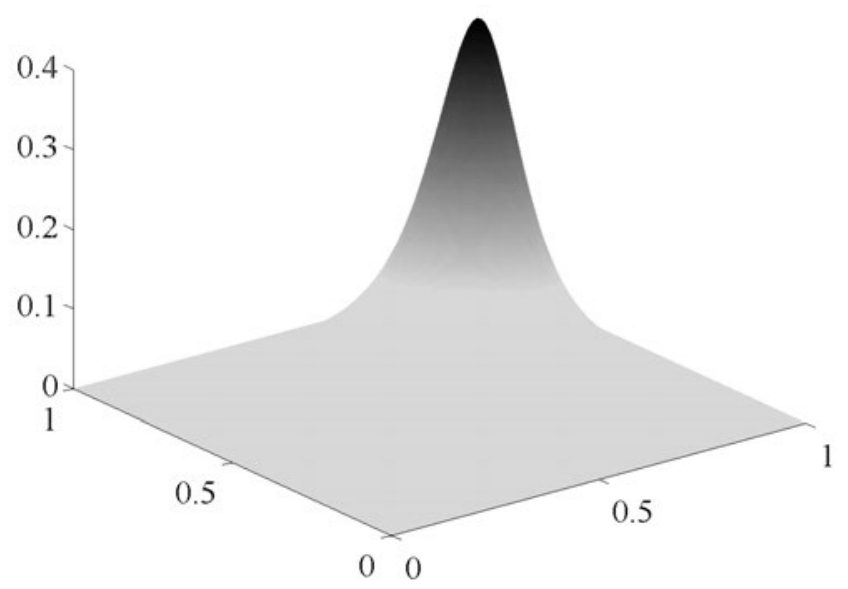

Figure 13. Example 2: analytical solution.

\begin{tabular}{|c|c|c|c|c|}
\hline$h$ & $l^{\mathcal{O}}\left(u_{H}\right)$ & $l^{\mathcal{O}}\left(u_{h}\right)$ & $a\left(e_{h}, \epsilon_{h}\right)$ & neglected terms \\
\hline \multirow{2nnnyy}{H}{$1 / 4$} & \multirow{3}{*}{$1.2510 \mathrm{E}-2$} & $1.3807 \mathrm{E}-2$ & $1.369 \mathrm{E}-3$ & $-7.2 \mathrm{E}-5$ \\
\cline { 1 - 4 }$H / 8$ & $1.4293 \mathrm{E}-2$ & $1.792 \mathrm{E}-3$ & $-9.0 \mathrm{E}-6$ \\
\cline { 1 - 1 } & & $1.4417 \mathrm{E}-2$ & $1.908 \mathrm{E}-3$ & $-1.0 \mathrm{E}-6$ \\
\hline
\end{tabular}

Table II. Example 2: comparison of the terms appearing in the representation of $l^{\mathcal{O}}\left(u_{h}\right)$ given in equation (6.1) for a fixed coarse particle distribution of $11 \times 11$ particles and for a different reference particle distributions.

The quantity of interest is the average of the solution over the domain.

As in the previous example, the influence of the different terms appearing in the representation for the output, $l^{\mathcal{O}}\left(u_{h}\right)$, is analyzed when reducing the size of the reference particle distribution $h$. The coarse particle distribution is a uniform distribution of $11 \times 11$ particles. The choice $h=H / 4$ yields to a difference between the neglected terms and $a\left(e_{h}, \epsilon_{h}\right)$ of more than one order of magnitude. It is worth noting that, while the neglected terms converge rapidly to zero as $h$ is decreased, the term $a\left(e_{h}, \epsilon_{h}\right)$ stabilized to the value of $a(e, \epsilon)$. Since the assumption made in Section 3.2 is valid, it is not necessary to use the alternative approach proposed in Section 4.3. In the following, the reference particle distribution is chosen such that $h=H / 4$. Thus, it is observed that the neglected terms affect the results only in their third significant digit.

The error estimation strategy and the computation of bounds is performed for a series of uniformly $H$-refined particle distributions, starting from an initial particle distribution of $5 \times 5$ particles and in each step dividing by 2 the interparticle distance $\left(H_{\text {new }}=H / 2\right)$. The resulting upper and lower bounds for $l^{\mathcal{O}}\left(e_{h}\right)$, shown in Figure 14, show the expected quadratic convergence rate.

A series of adapted particle distributions is produced. The initial and final particle 


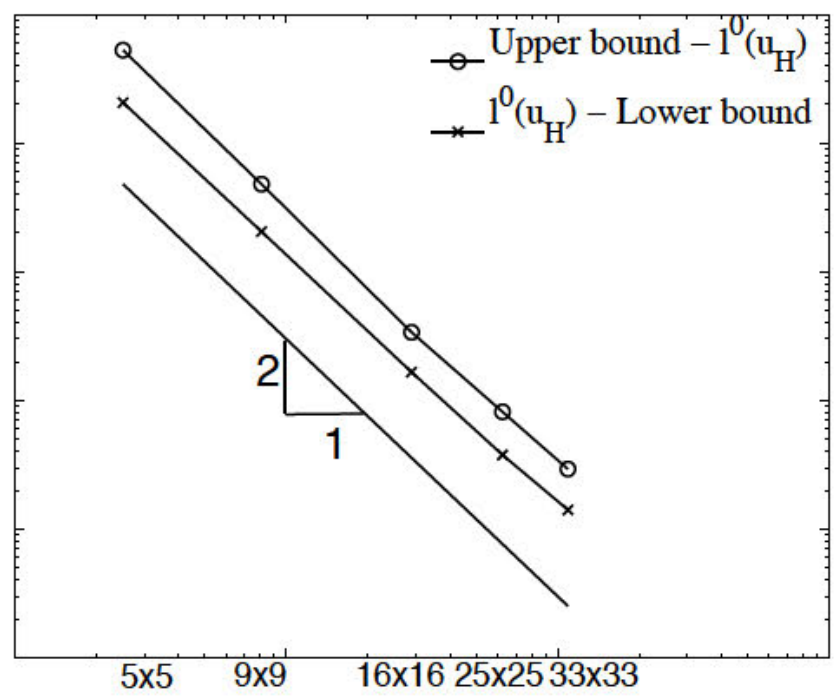

Figure 14. Example 2: convergence of the bounds for the error in the output $l^{\mathcal{O}}\left(e_{h}\right)$ for a uniform $H$-refinement of the particle distribution.

\#par=25

$$
\text { \#par=289 }
$$

Figure 15. Example 2: initial $(5 \times 5)$ and final $(289)$ distributions of particles in the adaptive procedure.

distributions are shown in Figure 15. Also the primal and adjoint EFG approximations of $u$ and $\psi$ are shown for the final particle distribution in Figure 16. For the initial coarse 

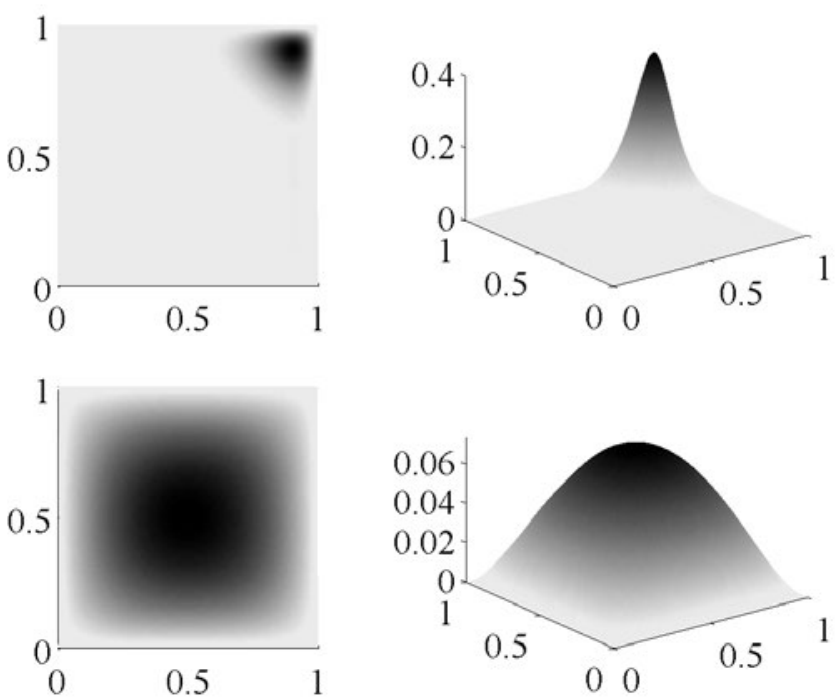

Figure 16. Example 2: primal and adjoint EFG approximations for the final particle distribution of the adaptive $H$-refinement (289 particles).

distribution of particles the output is bounded by, $l^{\mathcal{O}}\left(u_{h}\right) \in[-0.0097,0.0276]$, that is

$$
l^{\mathcal{O}}\left(u_{h}\right)=0.0090 \pm 0.0187=0.0090 \pm 207.78 \%,
$$

whereas, for the final distribution the output is bounded by $l^{\mathcal{O}}\left(u_{h}\right) \in[0.0143,0.0146]$

$$
l^{\mathcal{O}}\left(u_{h}\right)=0.0144 \pm 0.0002=0.0144 \pm 1.38 \% .
$$

Figure 17 shows the evolution of the lower bound, upper bound, bound average, EFG approximation and the exact output value in each step of the adaptive procedure.

Figure 18 shows the spatial distribution of the upper bound for the energy norm of the primal error, $\left\|e_{h}\right\|_{\mathrm{UB}}$. In this case this distribution is mainly conditioned by the original (primal) problem. This is because the adjoint problem associated with the quantity of interest $l^{\mathcal{O}}(\cdot)$ is pretty uniform, without zones of large gradient concentration.

\subsection{Example 3: convection-diffusion problem - solution with boundary and inner layers}

The proposed error estimation strategy to obtain bounds for quantities of interest is also tested for a convection-diffusion problem. The problem reads,

$$
\left\{\begin{aligned}
-\nu \nabla^{2} u+\mathbf{a} \cdot \nabla u=0 & & \text { in } \Omega=[0,1] \times[0,1], \\
u=1 & & \text { on } \Gamma_{1} \\
u=0 & & \text { on } \partial \Omega \backslash \bar{\Gamma}_{1} .
\end{aligned}\right.
$$

for a diffusion parameter $\nu=1.4 \cdot 10^{-2}$ and for a constant velocity field $\mathbf{a}=(\cos \pi / 6, \sin \pi / 6)$. The Dirichlet boundary conditions are chosen such that boundary and internal layers are present in the solution. Figure 19 shows the region $\Gamma_{1}$ where unit Dirichlet boundary conditions are imposed. The quantity of interest is the average of the solution over the whole domain. 


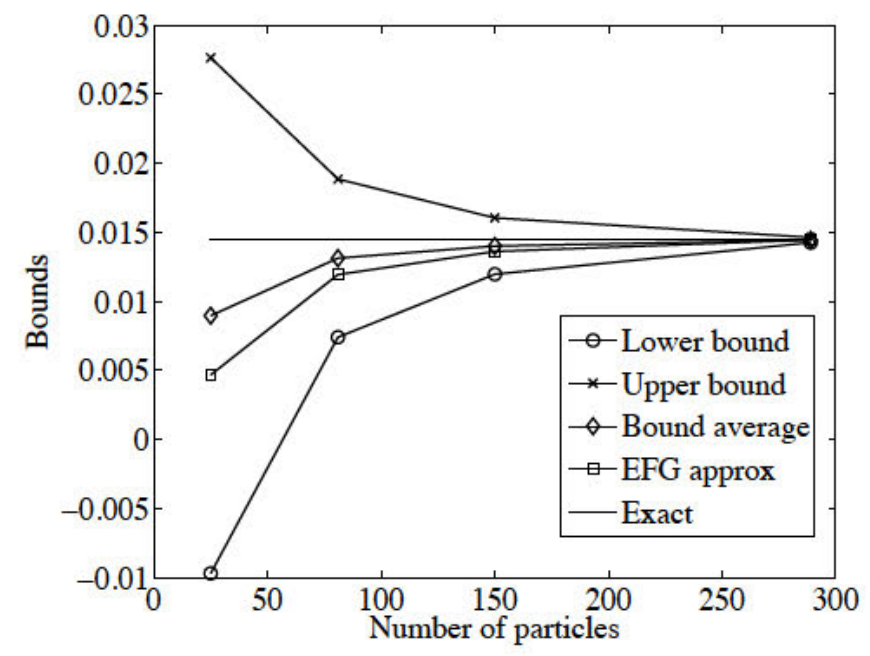

Figure 17. Example 2: estimates and bounds in the adaptive procedure.

Local error

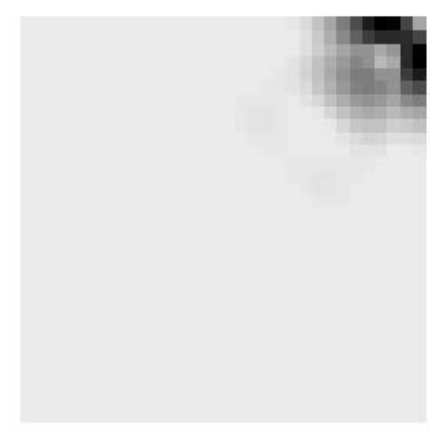

Local error

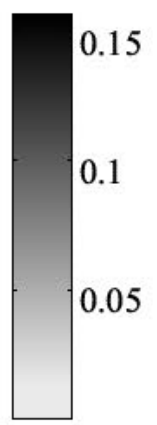

Figure 18. Example 2: local contributions to the upper bound of the energy norm of the primal error,

$\left\|e_{h}\right\|$, for the initial (left) and final (right) particle distribution of the adaptive $H$-refinement.

Remark 9. The error estimation strategy has been presented for the diffusion-reaction equation (see (1)). For this problem, the bilinear form $a(\cdot, \cdot)$ is symmetric and positive definite in $\mathcal{V}$, and therefore it defines a scalar product in $\mathcal{V}$. In a convection-diffusion problem, the associated bilinear form is no longer symmetric

$$
a(u, v)=\int_{\Omega} \boldsymbol{\nabla} v \cdot \nabla u+\mathbf{a} \cdot \boldsymbol{\nabla} u v d \Omega .
$$

Bounds for the output may be derived following [36] in a similar way as detailed in Section 3.4 introducing the symmetric counterpart of the bilinear form $a(w, v)$,

$$
a^{\mathrm{S}}(w, v)=\frac{1}{2}(a(w, v)+a(v, w))=\int_{\Omega} \nabla v \cdot \nabla u d \Omega .
$$




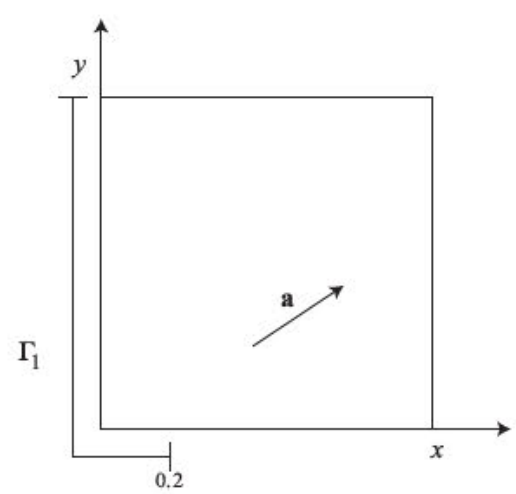

Figure 19. Example 3: boundary conditions for the model problem
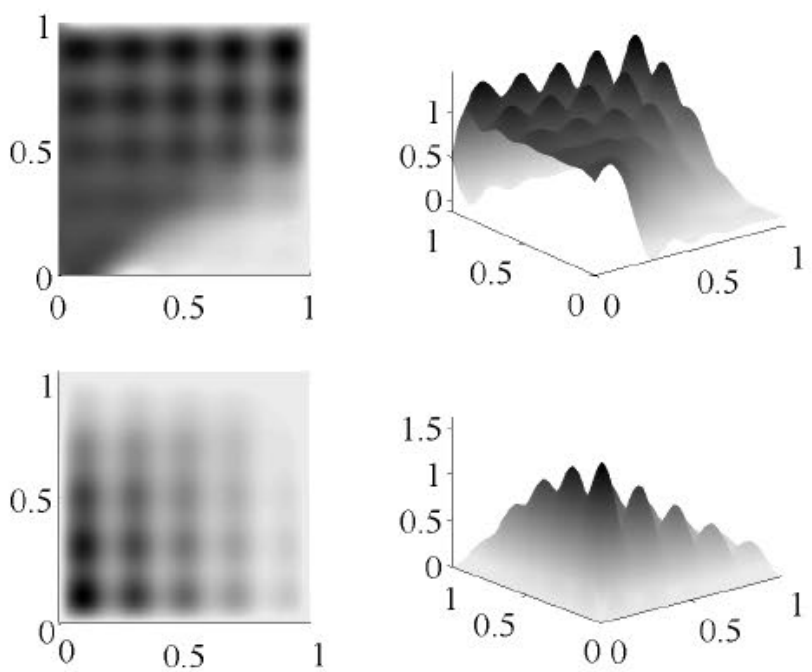

Figure 20. Example 3: primal and adjoint EFG approximations for the initial particle distribution $(21 \times 21$ particles $)$.

The only difference w.r.t. the procedure detailed in Figure 2, is that the local residual equations for $e_{\omega^{i}}^{\text {est }}$ and $\epsilon_{\omega^{i}}^{\text {est }}$ are computed using the restriction of the symmetric counterpart of the bilinear form to the star $\omega^{i}, a_{\omega^{i}}^{\mathrm{S}}(w, v)$, instead of $a_{\omega^{i}}(w, v)$. See [36] for a detailed description.

First, bounds for the output are computed for a uniform particle distribution of $11 \times 11$ particles. The primal and adjoint EFG approximations of $u$ and $\psi$ are shown in Figure 20. For this coarse particle distribution, the error estimation procedure yield a wide interval for the quantity of interest, $l^{\mathcal{O}}\left(u_{h}\right) \in[-0.2184,1.6237]$, that is

$$
l^{\mathcal{O}}\left(u_{h}\right)=0.7026 \pm 0.9210=0.7026 \pm 131.08 \%
$$


\#par=121

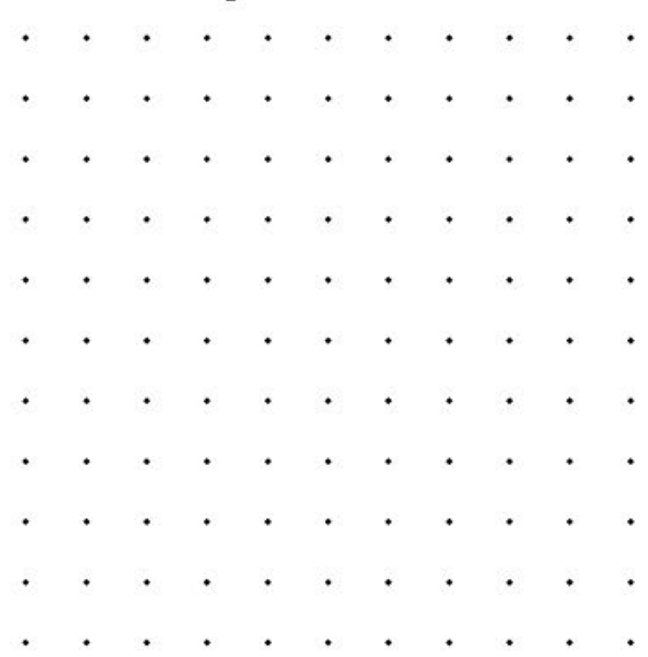

\#par=1035

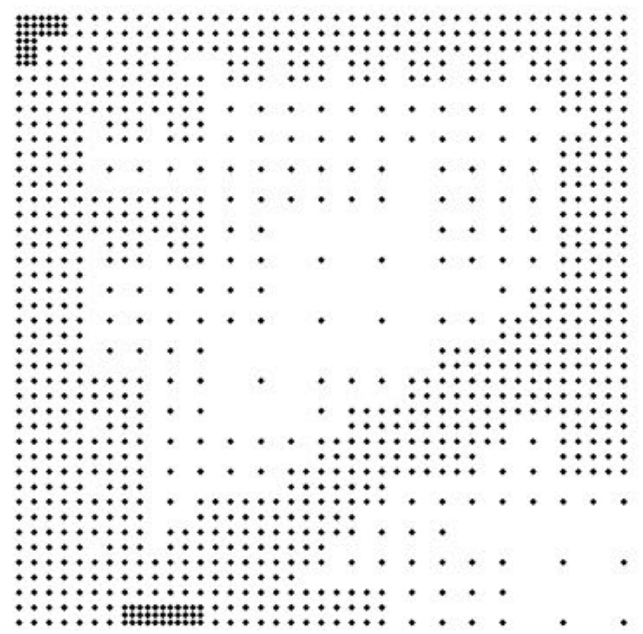

Figure 21. Example 3: initial $(11 \times 11)$ and final $(1035)$ distributions of particles for the adaptive procedure.

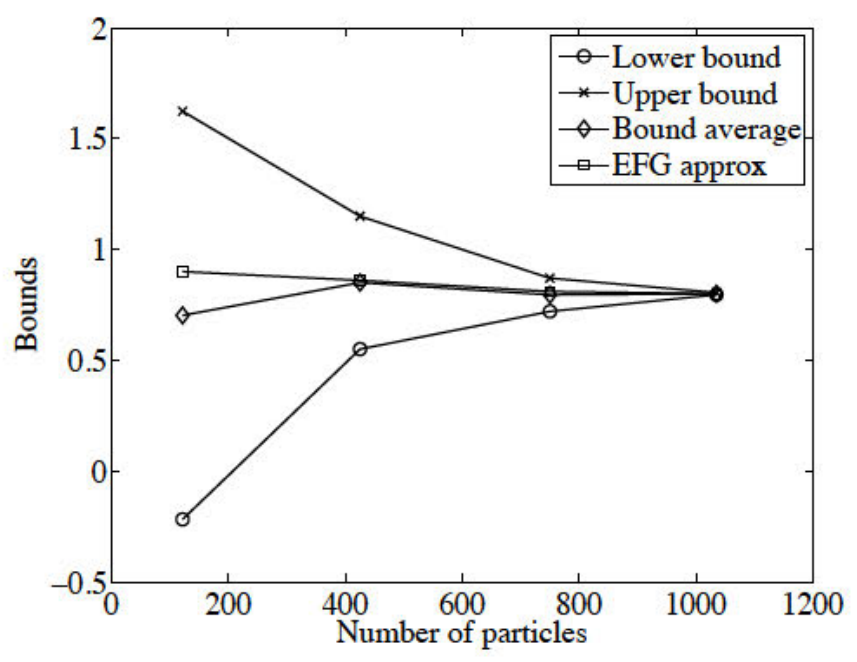

Figure 22. Example 3: bounds for an adaptive refinement strategy.

A series of adapted particle distributions are produced in order to reduce the initial half bound gap of $131.08 \%$. The goal being to reach a final gap of less than $2 \%$. The final distribution is shown in Figure 21 and produces the range, $l^{\mathcal{O}}\left(u_{h}\right) \in[0.8025,0.8224]$, that is

$$
l^{\mathcal{O}}\left(u_{h}\right)=0.8124 \pm 0.0099=0.8124 \pm 1.22 \% .
$$

The evolution of the bounds if shown in Figure 22, and the primal and adjoint EFG 

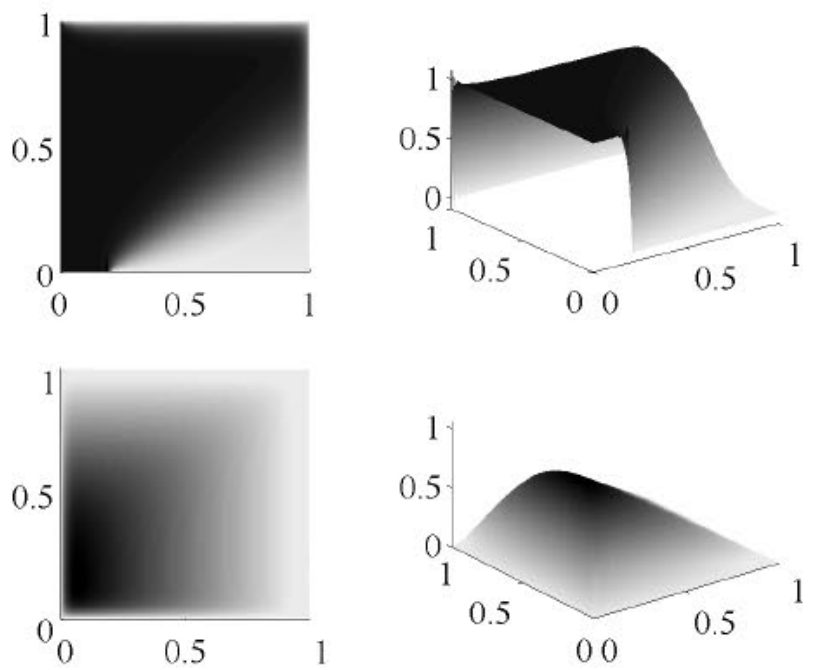

Figure 23. Example 3: primal and adjoint EFG approximations for the final particle distribution (1035 particles).

Local error

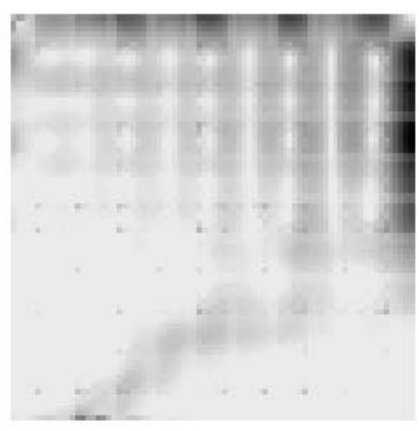

Local error

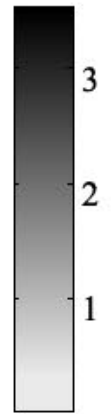

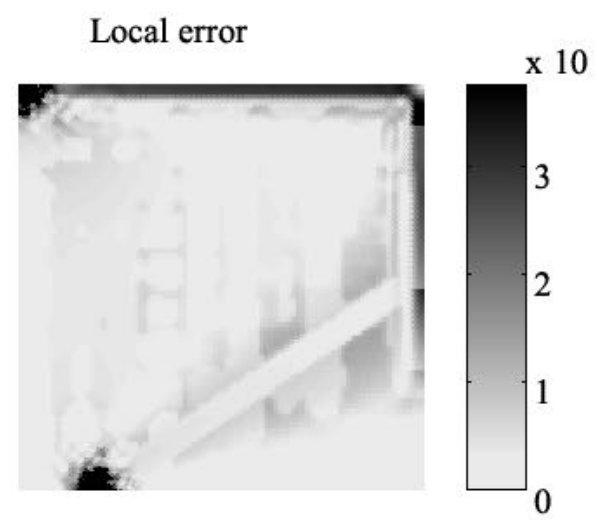

Figure 24. Example 3: local contributions to the upper bound of the energy norm of the primal error, $\left\|e_{h}\right\|$, for the initial (left) and final (right) particle distributions of the adaptive procedure.

approximations on the final particle distribution are shown in Figure 23. It can be seen that the adaptive procedure refines the particle distribution in the zones where the inner and boundary layer occur. Although no stabilization techniques are used, the final primal and adjoint EFG approximations present no oscillations.

Figure 24 shows the local behavior of the upper bound for the energy norm of the primal error both for the initial and final particle distributions.

Similarly to previous examples, the alternative approach proposed in Section 4.3 is not necessary. 


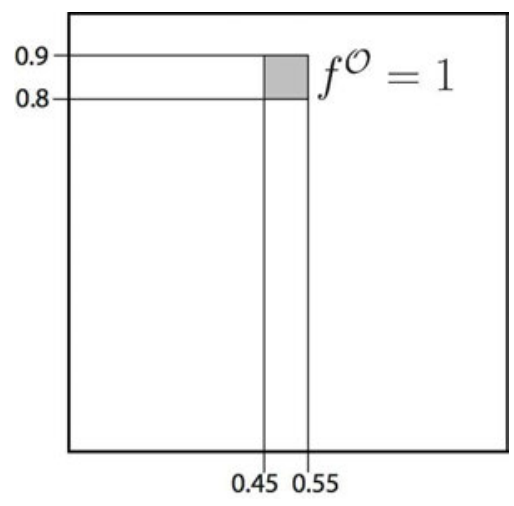

Figure 25. Example 4: rotating transport output region.

\subsection{Example 4: rotating transport}

The convection-diffusion equation is considered in the domain $\Omega=[0,1] \times[0,1]$ with homogeneous Dirichlet boundary conditions in all the boundary,

$$
-\nu \nabla^{2} u+\mathbf{a} \nabla u=s,
$$

for a diffusion parameter $\nu=10^{-2}$ and a divergence free velocity field $\mathbf{a}=(-y+0.5, x-0.5)$. The source term $s$ is

$$
s=\left\{\begin{array}{cc}
\cos \left(\frac{\pi}{2}\|\hat{x}\|\right) & \text { if }\|\hat{x}\| \leq 1 \\
0 & \text { otherwise }
\end{array}\right.
$$

where $\hat{x}=\frac{x-c}{\sigma}, c=(0.2,0.2), \sigma=0.2$.

The output of interest is a local average of the solution in the square region $[0.45,0.55] \times$ $[0.8,0.9]$, that is

$$
l^{\mathcal{O}}(u)=\int_{[0,1]^{2}} f^{\mathcal{O}} u d \Omega,
$$

where $f^{\mathcal{O}}=1$ in $[0.45,0.55] \times[0.8,0.9]$ and $f^{\mathcal{O}}=0$ elsewhere (see Figure 25).

The behavior of the bounds is analyzed starting with an initial particle distribution of $11 \times 11$ particles and adaptively refining the distribution. The initial and final particle distributions are shown in Figure 26, and the primal and adjoint EFG approximations of the exact solutions are shown in Figure 27 for the final particle distribution. As expected, the adaptive strategy refines both in the regions where either the primal and adjoint solutions present larger gradients.

The initial coarse distribution of particles predicts that the output is bounded by, $l^{\mathcal{O}}\left(u_{h}\right) \in$ $\left[3.312 \cdot 10^{-4}, 2.541 \cdot 10^{-3}\right]$, that is

$$
l^{\mathcal{O}}\left(u_{h}\right)=1.436 \cdot 10^{-3} \pm 1.105 \cdot 10^{-3}=1.436 \cdot 10^{-3} \pm 76.95 \%,
$$

whereas for the final distribution $l^{\mathcal{O}}\left(u_{h}\right) \in\left[1.168 \cdot 10^{-3}, 1.234 \cdot 10^{-3}\right]$,

$$
l^{\mathcal{O}}\left(u_{h}\right)=1.2 \cdot 10^{-3} \pm 3.3 \cdot 10^{-5}=1.2 \cdot 10^{-3} \pm 2.75 \% .
$$



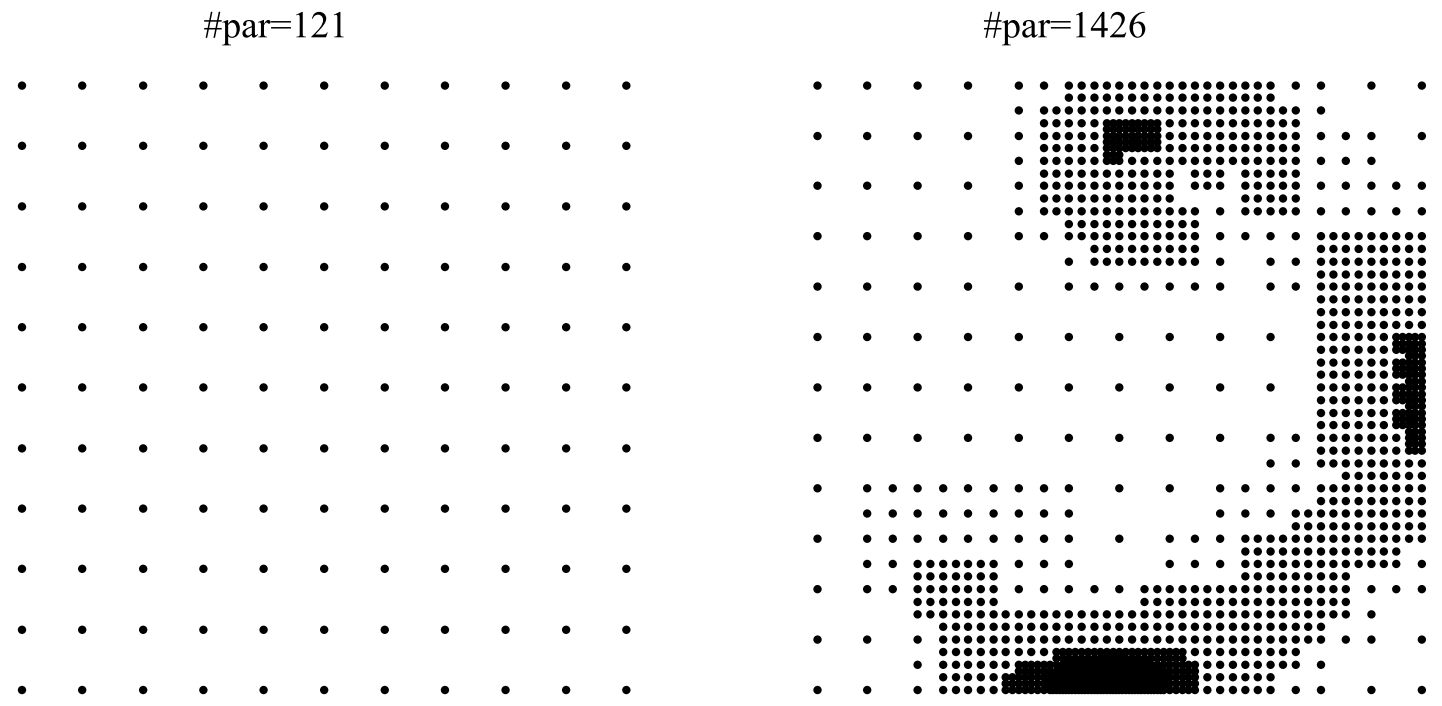

Figure 26. Example 4: initial $(11 \times 11)$ and final $(1426)$ distribution of particles for the adaptive refinement.
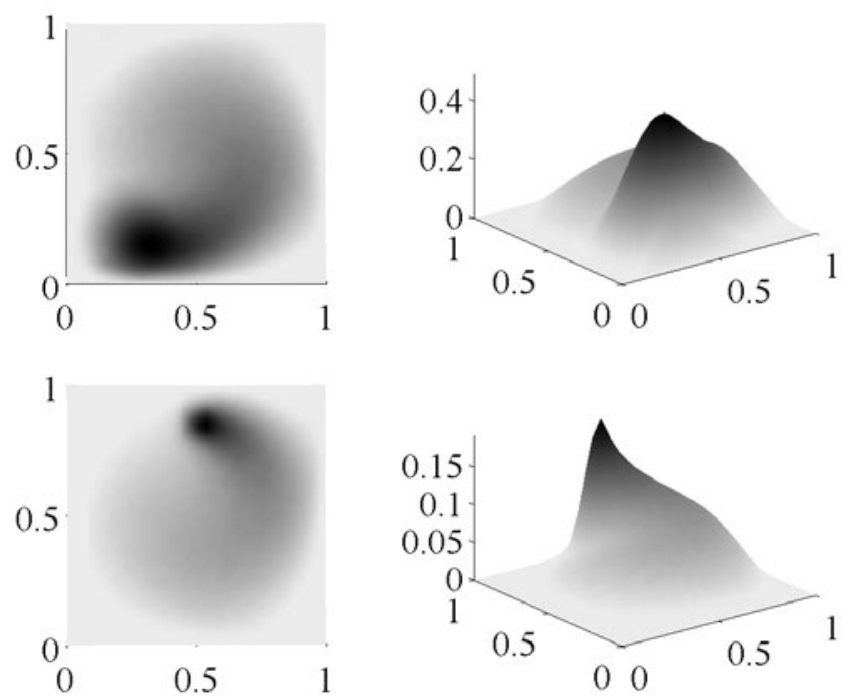

Figure 27. Example 4: primal and adjoint EFG approximations for the final particle distribution (1426 particles) of the adaptive refinement. 


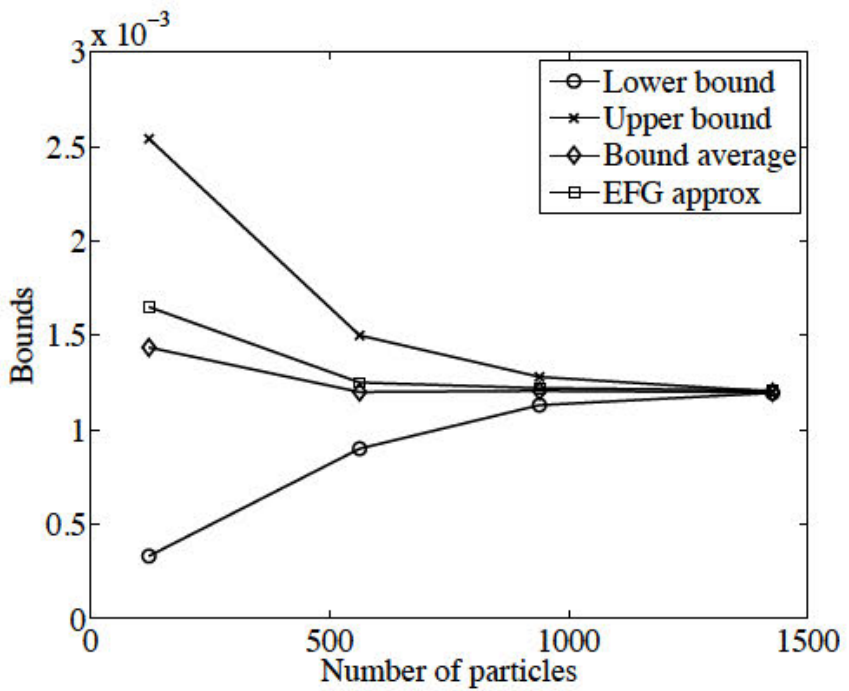

Figure 28. Example 4: computed bounds for an adaptive refinement.

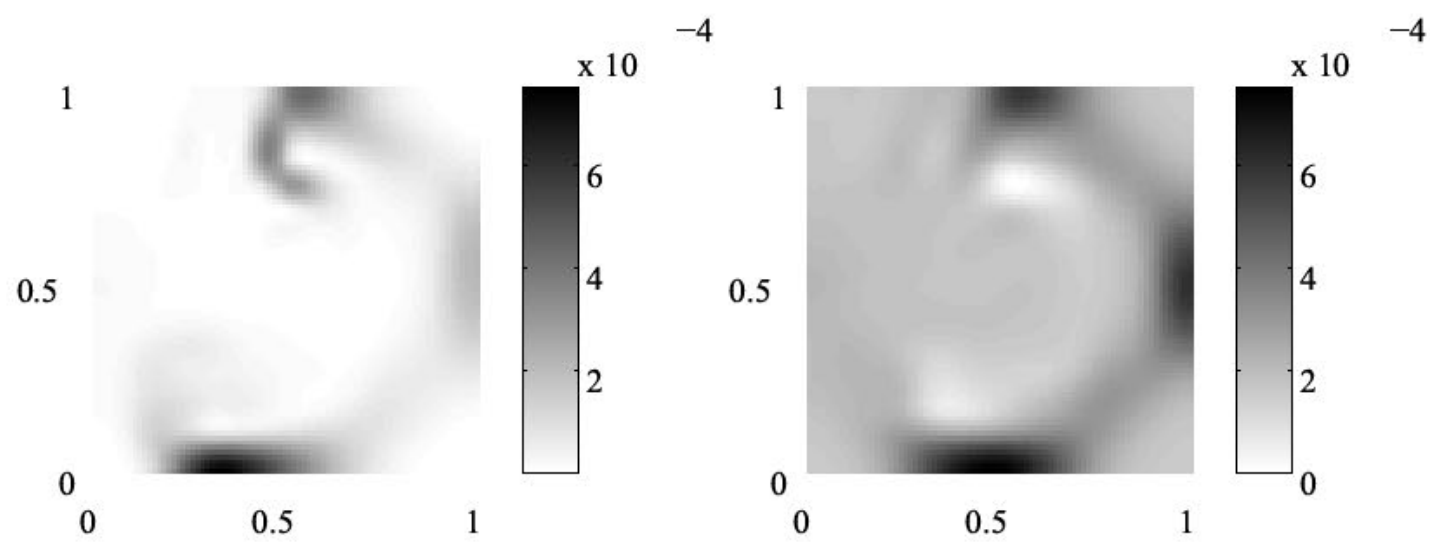

Figure 29. Example 4: spatial distribution of the half bound gap (left) and of $\left|l^{\mathcal{O}}\left(e_{h}\right)\right|$ (right).

The evolution of the bounds is shown in Figure 28. The convergence of the bounds is, as in the previous examples, quadratic as expected. However the resulting effectivities are not as good as in the two first examples. This is due to the fact that the used methodology yields poorer effectivities in highly convection-dominated problems. This behavior has also been noted in [36].

The spatial distribution of the half bound gap in the initial particle distribution is shown in Figure 29 along with the local contributions to $\left|l^{\mathcal{O}}\left(e_{h}\right)\right|$. As can be seen the local indicators provided by the half bound gap are in good agreement with the information provided by $\left|\ell_{k}^{\mathcal{O}}\left(e_{h}\right)\right|$.

Figure 30 shows the spatial distribution of the upper bound for the energy norm of the 

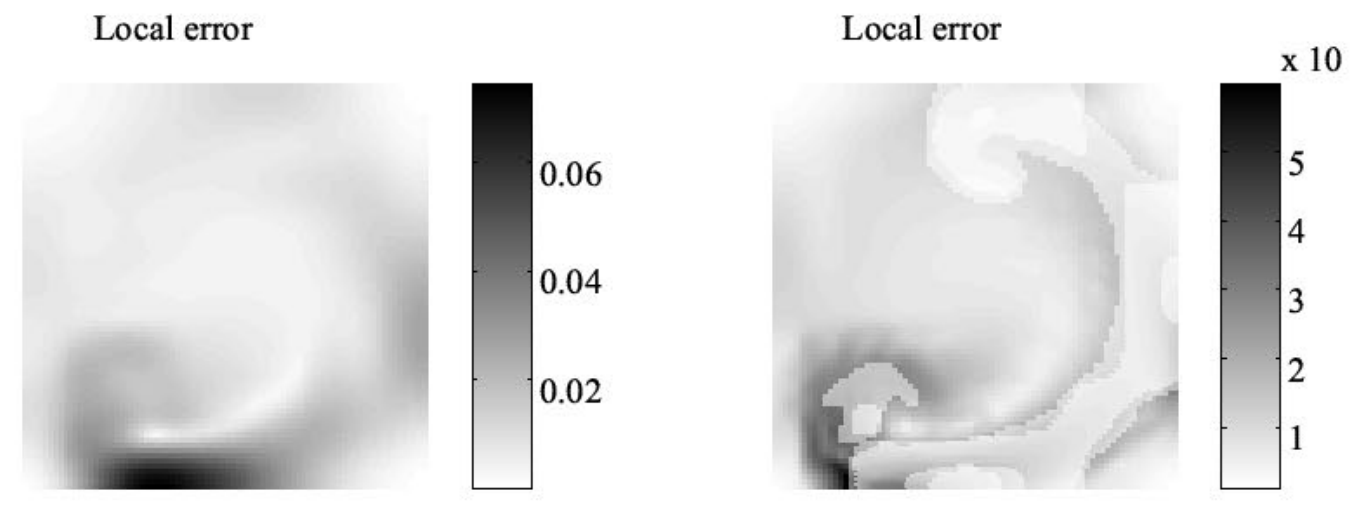

$-3$

Figure 30. Example 4: local contributions to the upper bound of the energy norm of the primal error, $\left\|e_{h}\right\|$, for the initial (left) and final (right) particle distribution of the adaptive refinement.

primal error, $\left\|e_{h}\right\|_{\mathrm{UB}}$, both for the initial and final particle distribution. Although the aim of the adaptive procedure is to reduce the error in the quantity of interest, it can be seen that the error in the energy norm of the primal problem is also drastically reduced.

Finally, the terms $a\left(e_{h}, \psi_{H}\right)$ and $l^{\mathcal{O}}\left(\bar{\pi}^{h} u_{H}-u_{H}\right)$ which are neglected in the computation of the bounds for the output - see equation (37) - have been computed also for this example for all the steps of the adaptive procedure. Adding this terms to the bounds for the output affect digits beyond the figures shown in the results. Consequently, the contribution of these terms is negligible and, as in the previous examples, it is not necessary to use the complementary error assessment proposed in Section 4.3.

\section{Conclusions}

An implicit residual-based error estimation strategy for meshfree methods is presented. The resulting estimator yields upper and lower bounds for outputs of interest. Moreover, the estimate has proved to be well suited to guide goal-oriented adaptive procedures. In this sense, a novel adaptive strategy has been devised to predict the desired interparticle size of the new discretization yielding a value of $\left|l^{\mathcal{O}}(e)\right|$ below some prescribed accuracy.

\section{REFERENCES}

1. M. Paraschivoiu, J. Peraire, and A. T. Patera. A posteriori finite element bounds for linear-functional outputs of elliptic partial differential equations. Comput. Methods Appl. Mech. Eng., 150(1-4):289-312, 1997. Symposium on Advances in Computational Mechanics, Vol. 2 (Austin, TX, 1997).

2. M. Ainsworth and J. T. Oden. A posteriori error estimation in finite element analysis. John Wiley \& Sons, Chichester, 2000.

3. J. T. Oden and S. Prudhomme. Goal-oriented error estimation and adaptivity for the finite element method. Comput. Math. Appl., 41(5-6):735-756, 2001.

4. I. Babuska and T. Strouboulis. The finite element method and its reliability. Numerical Mathematics and Scientific Computation. The Clarendon Press Oxford University Press, New York, 2001. 
5. J. Bonet, A. Huerta, and J. Peraire. The efficient computation of bounds for functionals of finite element solutions in large strain elasticity. Comput. Methods Appl. Mech. Engrg., 191(43):4807-4826, 2002.

6. A. M. Sauer-Budge, J. Bonet, A. Huerta, and J. Peraire. Computing bounds for linear functionals of exact weak solutions to Poisson's equation. SIAM J. Numer. Anal., 42(4):1610-1630, 2004.

7. N. Parés, J. Bonet, A. Huerta, and J. Peraire. The computation of bounds for linear-functional outputs of weak solutions to the two-dimensional elasticity equations. Comput. Methods Appl. Mech. Eng., 194(46):406-429, 2006.

8. N. Parés, P. Díez, and A. Huerta. Bounds of functional outputs for parabolic problems. Part I: Exact bounds of the discontinuous galerkin time discretization. Comput. Methods Appl. Mech. Eng. In press.

9. N. Parés, P. Díez, and A. Huerta. Bounds of functional outputs for parabolic problems. Part II: Bounds of the exact solution. Comput. Methods Appl. Mech. Eng. In press.

10. P. Ladevèze and D. Leguillon. Error estimate procedure in the finite element method and applications. SIAM J. Numer. Anal., 20(3):485-509, 1983.

11. C. Carstensen and S. A. Funken. Fully reliable localized error control in the FEM. SIAM J. Sci. Comput., 21(4):1465-1484 (electronic), 1999/00.

12. L. Machiels, Y. Maday, and A. T. Patera. A "flux-free" nodal Neumann subproblem approach to output bounds for partial differential equations. C. R. Acad. Sci. Paris Sér. I Math., 330(3):249-254, 2000.

13. P. Morin, R. H. Nochetto, and K. G. Siebert. Local problems on stars: a posteriori error estimators, convergence, and performance. Math. Comp., 72(243):1067-1097, 2003.

14. N. Parés, P. Díez, and A. Huerta. Subdomain-based flux-free a posteriori error estimators. Comput. Methods Appl. Mech. Eng., 195(4-6):297-323, 2006.

15. C. A. Duarte and J. T. Oden. An $h-p$ adaptive method using clouds. Comput. Methods Appl. Mech. Eng., 139(1-4):237-262, 1996.

16. W.K. Liu, S. Jun, D.T. Sihling, Y. Chen, and W. Hao. Multiresolution reproducing kernel particle method for computational fluid dynamics. Int. J. Numer. Methods Fluids, 24:1391-1415, 1997.

17. H.J. Chung and T. Belytschko. An error estimate in the EFG method. Comput. Mech., 21(2):91-100, 1998.

18. L. Gavete, J.L. Cuesta, and A. Ruiz. A procedure for approximation of the error in the EFG method. Int. J. Numer. Methods Eng., 53:667-690, 2002.

19. T. Strouboulis, L. Zhang, D. Wang, and I. Babuska. A posteriori error estimation for generalized finite element methods. Comput. Methods Appl. Mech. Engrg., 195(9-12):852-879, 2006.

20. Y. Maday, A. T. Patera, and J. Peraire. A general formulation for a posteriori bounds for output functionals of partial differential equations; application to the eigenvalue problem. $C$. R. Acad. Sci. Paris Sér. I Math., 328(9):823-828, 1999.

21. S. Prudhomme and J. T. Oden. On goal-oriented error estimation for elliptic problems: application to the control of pointwise errors. Comput. Methods Appl. Mech. Eng., 176(1-4):313-331, 1999. New advances in computational methods (Cachan, 1997).

22. A. T. Patera and J. Peraire. A general Lagrangian formulation for the computation of a posteriori finite element bounds. In Error estimation and adaptive discretization methods in computational fluid dynamics, volume 25 of Lect. Notes Comput. Sci. Eng., pages 159-206. Springer, Berlin, 2003.

23. Z.C. Xuan, N. Parés, and J. Peraire. Computing upper and lower bounds for the J-integral in twodimensional linear elasticity. Comput. Methods Appl. Mech. Eng., 195(4-6):430-443, 2006.

24. S. Fernández-Méndez and A. Huerta. Imposing essential boundary conditions in mesh-free methods. Comput. Methods Appl. Mech. Eng., 193(12-14):1257-1275, 2004.

25. A. Huerta and S. Fernández-Méndez. Enrichment and coupling of the finite element and meshless methods. Int. J. Numer. Methods Eng., 48(11):1615-1636, 2000.

26. A. Huerta, S. Fernández-Méndez, and W. K. Liu. A comparison of two formulations to blend finite elements and mesh-free methods. Comput. Methods Appl. Mech. Eng., 193(12-14):1105-1117, 2004.

27. S. Fernández-Méndez, J. Bonet, and A. Huerta. Continuous blending of SPH with finite elements. Comput. Struct., 83(17-18):1448-1458, 2005.

28. J. Nitsche. über ein variations zur lösung von dirichlet-problemen bei verwendung von teilräumen die keinen randbedingungen unterworfen sind. Abh. Math. Se. Univ., 36:9-15, 1970.

29. R. Stenberg. On some techniques for approximating boundary conditions in the finite element method. J. Comput. Appl. Math., 63(1-3):139-148, 1995.

30. R. Becker. Mesh adaptation for Dirichlet flow control via Nitsche's method. Commun. Numer. Methods Eng., 18(9):669-680, 2002.

31. M. Griebel and M. A. Schweitzer. A particle-partition of unity method. Part V: Boundary conditions. In S. Hildebrandt and H. Karcher, editors, Geometric Analysis and Nonlinear Partial Differential Equations, pages 517-540, Berlin, 2002. Springer.

32. P. Díez and G. Calderón. Remeshing criteria and proper error representations for goal oriented hadaptivity. Comput. Methods Appl. Mech. Eng., 196(4-6):719-733, 2007. 
33. W. K. Liu, S. Li, and T. Belytschko. Moving least square reproducing kernel methods Part I: Methodology and convergence. Comput. Methods Appl. Mech. Eng., 143(1-2):113-154, 1997.

34. E. Oñate and G. Bugeda. A study of mesh optimality criteria in adaptative finite element analysis. Engrg. Comput., 10:307-321, 1993.

35. P. Díez, N. Parés, and A. Huerta. Recovering lower bounds of the error by postprocessing implicit residual a posteriori error estimates. Internat. J. Numer. Methods Engrg., 56(10):1465-1488, 2003.

36. M. Paraschivoiu, J. Peraire, and A. T. Patera. A posteriori finite element bounds for linear-functional outputs of elliptic partial differential equations. Comput. Methods Appl. Mech. Engrg., 150(1-4):289-312, 1997. Symposium on Advances in Computational Mechanics, Vol. 2 (Austin, TX, 1997). 